\title{
Broken ergodicity in two-dimensional homogeneous magnetohydrodynamic turbulence
}

\author{
John V. Shebalin \\ Astromaterials Research $\&$ Exploration Science Office \\ NASA Johnson Space Center, Houston, Texas 77058-3696
}

\begin{abstract}
Two-dimensional (2-D) homogeneous magnetohydrodynamic (MHD) turbulence has many of the same qualitative features as three-dimensional (3-D) homogeneous MHD turbulence. These features include several ideal invariants, along with the phenomenon of broken ergodicity. Broken ergodicity appears when certain modes act like random variables with mean values that are large compared to their standard deviations, indicating a coherent structure or dynamo. Recently, the origin of broken ergodicity in 3-D MHD turbulence that is manifest in the lowest wavenumbers was explained. Here, a detailed description of the origins of broken ergodicity in 2-D MHD turbulence is presented. It will be seen that broken ergodicity in ideal 2-D MHD turbulence can be manifest in the lowest wavenumbers of a finite numerical model for certain initial conditions or in the highest wavenumbers for another set of initial conditions. The origins of broken ergodicity in ideal 2-D homogeneous MHD turbulence are found through an eigenanalysis of the covariance matrices of the modal probability density functions. It will also be shown that when the lowest wavenumber magnetic field becomes quasi-stationary, the higher wavenumber modes can propagate as Alfvén waves on these almost static large-scale magnetic structures.

PACS numbers: 05.20.Jj, 47.27.Gs, 91.25.Cw, 95.30.Qd
\end{abstract}

Keywords: Statistical Mechanics, Turbulence, Dynamo, Magnetohydrodynamics 


\section{INTRODUCTION}

Turbulence in conducting fluids is a universal phenomenon occurring in stars, stellar winds, planetary interiors and magnetospheres, as well as in magnetic fusion energy devices and in the liquid metal cooling systems of fission reactors. Understanding such magnetohydrodynamic (MHD) turbulence is of great importance because of the enhanced small-scale transport it affords when compared to molecular viscosity, resistivity and thermal conduction, in addition to the processes of large-scale self-organization it enables through its inherent nonlinearity. Although much progress has been made ${ }^{1}$, the study of turbulence is still a challenging endeavor, due to the vast number of independent variables that must be modeled or measured, which may be characterized as turbulent eddies and magnetic flux tubes, or as Fourier modes corresponding to length scales ranging from the size of the confining system to the dissipation scale at which nonlinear effects are no longer important. As is well known, the effective number of degrees-of-freedom in three-dimensional (3-D) flows is proportional to Reynolds number to the $9 / 4$ power and in two-dimensions (2-D), it is proportional to Reynolds number squared ${ }^{2}$. Although this number is finite, it is large enough that numerical simulation of turbulent systems remains very challenging.

Turbulence is thus a problem of statistical physics ${ }^{3,4}$ and a firm basis from which to begin understanding MHD turbulence is equilibrium statistical mechanics ${ }^{5,6}$, which, by its nature, requires us to consider non-dissipative, conservative model systems. Although a gas of atoms or molecules may have only one essential conserved quantity (the total energy), in the case of ideal (that is, non-dissipative) homogeneous MHD turbulence there are three global invariants ${ }^{5,6}$ (unless a constant external mean magnetic field is imposed, in which

case it has two invariants ${ }^{7}$ ). A gas, with only one ideal invariant, is expected to have this energy equipartioned over its degrees-of-freedom, while the presence of more than one ideal invariant in either 2-D or 3-D MHD turbulence leads to a statistical distribution of energy that may strongly favor a small fraction of available modes ${ }^{5,6}$. These favored modes are at the largest length scales in 3-D and may be at either the largest or the smallest length scales in models of ideal 2-D MHD turbulence, as will be seen. When these favored modes have sufficiently more energy than other modes, broken ergodicity (i.e., apparently nonergodic behavior that does not disappear on reasonably long timescales ${ }^{8}$ ) occurs and the magnetofluid 'self-organizes' so that these modes appear to behave like random variables 
with large mean values and relatively small standard deviations ${ }^{9}$. In other words, what may be called a magnetic dynamo or coherent structure occurs naturally in ideal MHD turbulence due to the process of 'broken ergodicity'.

Broken ergodicity and its relation to magnetic dynamos and coherent structure was discussed recently in great detail for 3-D homogeneous MHD turbulence, where eigenanalysis was used to discover the essence of the phenomena ${ }^{10}$. Here, we focus on ideal 2-D homogeneous MHD turbulence and show that the 2-D case contains novel features not found in the 3-D case. We study a finite Fourier model of 2-D MHD turbulence and determine the eigenvariables and eigenvalues associated with the modal Hermitian $2 \times 2$ covariance matrices appearing in the probability density function. This allows us to analyze data from numerical experiments and uncover the underlying structure of 2-D ideal MHD turbulence, and also to observe the propagation of higher wavenumber Alfvén waves on quasi-static lowestwave number magnetic fields even when there is no mean magnetic field present. Also, although Fourier models have periodic boundaries, these can, serve as surrogates of physical confinement ${ }^{11-13}$, allowing an investigation of large-scale behavior. The restriction to a 2-D planar geometry both reflects the observed 2-D behavior of plasmas in relatively strong external magnetic fields ${ }^{14,15}$ and allows access to much higher wavenumbers for long-time numerical simulations than are possible in 3-D simulations. In this paper, ideal 2-D MHD turbulence is of primary concern and although broken ergodicity in dissipative MHD turbulence can also be studied using 2-D models, which could be very informative even though 3-D models are more realistic, this will be deferred for now.

\section{BASIC EQUATIONS}

Here, a turbulent magnetofluid will be assumed to flow only in the $x-y$ plane, with position vectors $\mathbf{x}=x \hat{\mathbf{x}}+y \hat{\mathbf{y}}$, where $\hat{\mathbf{x}}$ and $\hat{\mathbf{y}}$ provide an orthonormal basis and where $\hat{\mathbf{z}}$ is a unit vector normal to this plane, so that $\hat{\mathbf{z}} \cdot \hat{\mathbf{x}} \times \hat{\mathbf{y}}=1$. The non-dimensional form of the $2-\mathrm{D}$

incompressible MHD equations are well known ${ }^{1}$, and may be written as (with $\partial_{t} \equiv \partial / \partial t$, etc.),

$$
\begin{aligned}
& \partial_{t} \omega=Z(j, a)+Z(\psi, \omega)+\mathbf{B}_{\mathrm{o}} \cdot \nabla j+\nu \nabla^{2} \omega, \\
& \partial_{t} a=Z(\psi, a)+\mathbf{B}_{\mathrm{o}} \cdot \nabla \psi+\eta \nabla^{2} a .
\end{aligned}
$$


The Jacobian $Z(f, g)$ contains the nonlinear interactions and has the form

$$
Z(f, g) \equiv \partial_{x} f \partial_{y} g-\partial_{y} f \partial_{x} g
$$

The vorticity $\omega(\mathbf{x}, t)$ is related to the stream function $\psi(\mathbf{x}, t)$ by $\omega=-\nabla^{2} \psi$, and the electric current $j(\mathbf{x}, t)$ is related to the magnetic potential $a(\mathbf{x}, t)$ by $j=-\nabla^{2} a$. The magnetofluid velocity is $\mathbf{u}=\nabla \times \hat{\mathbf{z}} \psi$, the magnetic induction is $\mathbf{b}=\nabla \times \hat{\mathbf{z}} a$, and these clearly satisfy $\nabla \cdot \mathbf{u}=\nabla \cdot \mathbf{b}=0$, where $\nabla=\hat{\mathbf{x}} \partial_{x}+\hat{\mathbf{y}} \partial_{y}$. Density does not appear in (1) because it equals unity, $\nu$ in (1) is the kinematic viscosity, while $\eta$ in (2) is the magnetic diffusivity. The variables $\psi(\mathbf{x}, t), a(\mathbf{x}, t)$ and their derivatives are assumed to be periodic within a square of edge length $2 \pi$; this periodic square can be used to represent a small region within a much larger area of homogeneous flow or as a surrogate for a bounded physical area (or as a manifold without boundary). In eqs. 1 and $2, \mathbf{B}_{\mathrm{o}}$ is the constant, mean magnetic field (which can be zero).

Here, we assume periodic boundary conditions on a square with side length $2 \pi$. Let us define the spatial average of a quantity $f(\mathbf{x}) g(\mathbf{x})$ as $\widehat{f g}$, where

$$
\widehat{f g} \equiv \frac{1}{4 \pi^{2}} \int_{0}^{2 \pi} d y \int_{0}^{2 \pi} d x f(\mathbf{x}) g(\mathbf{x})
$$

Spatial averages of the energy $\widehat{E}$, cross helicity $\widehat{H_{C}}$ and mean squared magnetic potential $\widehat{A}$, as well as the enstrophy $\widehat{\Omega}$ and mean square current $\widehat{J}$, are defined by

$$
\begin{aligned}
\widehat{E} & =\frac{1}{2}(\widehat{\psi \omega}+\widehat{a j})=\frac{1}{2}\left(\widehat{u^{2}}+\widehat{b^{2}}\right), \\
\widehat{H_{C}} & =\frac{1}{2} \widehat{a \omega}=\frac{1}{2} \widehat{\mathbf{u} \cdot \mathbf{b}}=\frac{1}{2} \widehat{j \psi}, \\
\widehat{A} & =\frac{1}{2} \widehat{a^{2}}, \quad \widehat{\Omega}=\frac{1}{2} \widehat{\omega^{2}}, \quad \widehat{J}=\frac{1}{2} \widehat{j^{2}} .
\end{aligned}
$$

We define these averages first, rather than the total quantities, because these averages are independent of numerical grid size. Initial conditions are generally chosen so that $\widehat{E}=1$, as is appropriate for the dimensionless equations (1) and (2). On an $N \times N$ numerical grid, the total energy is $E=N^{2} \widehat{E}$, and similarly $H_{C}=N^{2} \widehat{H_{C}}, A=N^{2} \widehat{A}$, etc. Spatial grid-point averages are useful in that they allow a more straightforward comparison between simulations with different values of $N$. 
Using equations (1) and (2), it is straightforward to find

$$
\begin{aligned}
\frac{d \widehat{E}}{d t} & =-2(\nu \widehat{\Omega}+\eta \widehat{J}), \\
\frac{d \widehat{H_{C}}}{d t} & =-\frac{1}{2}(\nu+\eta) \widehat{j \omega}, \\
\frac{d \widehat{A}}{d t} & =\mathbf{B}_{\mathrm{o}} \times \hat{\mathbf{z}} \cdot \widehat{a \mathbf{u}}-\eta \widehat{b^{2}} .
\end{aligned}
$$

If we have $\nu=\eta=0$, then $\widehat{E}$ and $\widehat{H_{C}}$ are constants of the motion, and if $\mathbf{B}_{\mathrm{o}}=0$, then $\widehat{A}$ is also constant. In de-aliased Fourier models of 2-D MHD turbulence, it can be shown that these three quadratic forms are the only constants of the motion ${ }^{16}$.

Briefly, consider the case where $\mathbf{B}_{\mathrm{o}}=0$ and $\eta=0$, and the equation (2) takes the form

$$
\frac{d a}{d t}=\frac{\partial a}{\partial t}+\mathbf{u} \cdot \nabla a=0
$$

This indicates that the value of $a$ for every moving fluid point in an ideal 2-D magnetofluid is a constant of the motion. However, to capture this property, we must adopt a Lagrangian approach and use a numerical method that tracks individual points. Instead, the standard procedure in homogeneous MHD turbulence simulations is to follow an Eulerian approach that uses a Fourier spectral transform method ${ }^{17}$, a method based on the time-dependent values of $a$ (and $\omega$ ) on a fixed, regularly spaced, finite grid of points. This Eulerian approach does not allow the tracking of individual fluid elements, so, in general, the Lagrangian invariants $a(\mathbf{x}(t))$ associated with each fluid element play no role in the Fourier method. Although a Lagrangian approach that tracks individual vortex lines exists for ideal 2-D fluid mechanics ${ }^{18}$, where the governing equation is $(11)$ with $a$ replaced by $\omega(i . e ., d \omega / d t=0)$, and for the analogous 2-D motion of parallel electric line charges ${ }^{19}$, there appears to be no similar approach possible in ideal 2-D MHD, as this would require $d \omega / d t=0$ and $d j / d t=0$, and neither of these are the equations of 2-D MHD.

\section{FOURIER MODELS}

The system we employ to model 2-D MHD turbulence uses the finite Fourier series corresponding to $\omega$ and $a$. The physical fields $\omega(\mathbf{x})$ and $a(\mathbf{x})$ in $\mathbf{x}$-space are connected to their 
corresponding Fourier coefficients $\tilde{\omega}(\mathbf{k})$ and $\tilde{a}(\mathbf{k})$ in k-space by transformations of the form (where $i \equiv \sqrt{-1}$ )

$$
f(\mathbf{x}, t)=\frac{1}{N} \sum_{\mathbf{k} \in \mathcal{K}} \tilde{f}(\mathbf{k}, t) e^{i \mathbf{k} \cdot \mathbf{x}}, \quad \tilde{f}(\mathbf{k}, t)=\frac{1}{N} \sum_{\mathbf{x} \in \mathcal{P}} f(\mathbf{x}, t) e^{-i \mathbf{k} \cdot \mathbf{x}} .
$$

The sums are over discrete sets $\mathcal{K}$ and $\mathcal{P}$. The set $\mathcal{K}$ contains those wave vectors (i.e., k-space grid points) $\mathbf{k}$ that have integer components $k_{x}=k_{1}$ and $k_{y}=k_{2}$, whose values lie between $-N / 2+1$ and $N / 2 ; \mathcal{K}$ thus has $N^{2}$ elements. The set $\mathcal{P}$ consists of position vectors (i.e., $\mathrm{x}$-space grid points) $\mathrm{x}$ that have components $x_{j}=2 \pi m_{j} / N\left(j=1,2 ; 0 \leq m_{j}<N\right)$, with $x=x_{1}, y=x_{2}, m_{x}=m_{1}$, and $m_{y}=m_{2}$, where the $m_{j}$ are integers; $N$ is the number of grid points in each dimension and the set $\mathcal{P}$ also has $N^{2}$ elements.

Since $\mathbf{x}$-space variables, such as $a(\mathbf{x})$ are real, their Fourier transforms satisfy relations of the form $\tilde{a}(-\mathbf{k})=\tilde{a}^{*}(\mathbf{k})$, where ${ }^{(*}$ ' indicates complex conjugation. The $\tilde{a}(\mathbf{k})$ have real and imaginary parts $\tilde{a}_{R}(\mathbf{k})$ and $\tilde{a}_{I}(\mathbf{k})$, so that $\tilde{a}(\mathbf{k})=\tilde{a}_{R}(\mathbf{k})+i \tilde{a}_{I}(\mathbf{k})$, which implies

$$
\tilde{a}_{R}(-\mathbf{k})=\tilde{a}_{R}(\mathbf{k}), \quad \tilde{a}_{I}(-\mathbf{k})=-\tilde{a}_{I}(\mathbf{k}) .
$$

Thus, only about half of the $\tilde{a}(\mathbf{k})$ for $\mathbf{k} \in \mathcal{K}$ are independent, i.e., those corresponding to $\mathbf{k}$, but not to $-\mathbf{k}$. Now, each $\tilde{a}(\mathbf{k}), \mathbf{k} \in \mathcal{K}$, generally has one real and one imaginary component, i.e., except for coefficients with $\mathbf{k}=\left(k_{x}, k_{y}\right)=(0,0),(0, N / 2),(N / 2,0)$ and $(N / 2, N / 2)$, which only have real components. Nevertheless, a careful count shows that the set of coefficients $\tilde{a}(\mathbf{k}), \mathbf{k} \in \mathcal{K}$, also have a $N^{2}$ independent parts, before any further restrictions are imposed.

However, these excepted coefficients are not used as dynamical variables in Fourier method solutions of equations (1) and (2), because only Fourier coefficients with $k=|\mathbf{k}|$ such that $1 \leq k \leq K<N / 2$ are allowed to be nonzero. Coefficients with wavevectors $\mathbf{k}$ outside the 'isotropic truncation radius' $K$, or at $k=0$, will always be set to zero. (Here, we use $K=\sqrt{2} N / 3$, a value set by the de-aliasing procedure ${ }^{20}$ we employ.) The largest dynamical scale in the periodic box is represented by the $k=1$ modes and although $\mathcal{K}$ contains a total of $N^{2}$ wavevectors, only $\mathcal{N} \cong \pi K^{2}$ vectors $\mathbf{k}$ satisfy $1 \leq k \leq K$ and participate in the dynamical evolution of the model system. For convenience, let us define the set $\mathcal{K}^{\prime} \subset \mathcal{K}$ by

$$
\mathcal{K}^{\prime}=\left\{\mathbf{k} \mid 1 \leq k \leq K \text {; if } \mathbf{k} \in \mathcal{K}^{\prime} \text {, then }-\mathbf{k} \notin \mathcal{K}^{\prime}\right\}
$$

The set $\mathcal{K}^{\prime}$ has $\mathcal{N}^{\prime}=\mathcal{N} / 2$ elements. If we have a set $\mathcal{K}^{\prime}$, an equivalent set can be created by removing $\mathbf{k}$ from $\mathcal{K}^{\prime}$ and replacing it by $-\mathbf{k}$; there are obviously $2^{\mathcal{N}^{\prime}}$ possible equivalent 
choices for $\mathcal{K}^{\prime}$. The exact choice depends on the particular computer code we use; in what follows, for $\mathbf{k}=\left(k_{x}, 0\right)$, we allow $1 \leq k_{x} \leq K$; and for $\mathbf{k}=\left(k_{x}, k_{y}\right)$, we allow $1 \leq k_{y} \leq K$ and $-K \leq k_{x} \leq K$, so long as $1 \leq\left(k_{x}^{2}+k_{y}^{2}\right) \leq K$.

Using (12), it can easily be shown that for any pair of variables $f$ and $g$,

$$
\sum_{\mathbf{x} \in \mathcal{P}} f(\mathbf{x}, t) g(\mathbf{x}, t)=\sum_{\mathbf{k} \in \mathcal{K}} \tilde{f}^{*}(\mathbf{k}, t) \tilde{g}(\mathbf{k}, t)
$$

In addition, the global average (4) can now be expressed as

$$
\widehat{f g}=\frac{1}{N^{2}} \sum_{\mathbf{k} \in \mathcal{K}} \tilde{f}^{*}(\mathbf{k}, t) \tilde{g}(\mathbf{k}, t)
$$

In what follows, we often omit $t$ from the argument of the coefficients.

Performing a Fourier transform on the equations (1) and (2), yields a finite, autonomous dynamical system:

$$
\begin{aligned}
& \frac{d \tilde{\omega}(\mathbf{k})}{d t}=\{Z(j, a)\}_{\mathbf{k}}+\{Z(\psi, \omega)\}_{\mathbf{k}}+i \mathbf{B}_{\mathrm{o}} \cdot \mathbf{k} \tilde{j}(\mathbf{k})-\nu k^{2} \tilde{\omega}(\mathbf{k}) \\
& \frac{d \tilde{a}(\mathbf{k})}{d t}=\{Z(\psi, a)\}_{\mathbf{k}}+i \mathbf{B}_{\mathrm{o}} \cdot \mathbf{k} \tilde{\psi}(\mathbf{k})-\eta k^{2} \tilde{a}(\mathbf{k}) .
\end{aligned}
$$

Here, we use (12) to transform the relations $\omega=-\nabla^{2} \psi$ and $j=-\nabla^{2} a$ into k-space:

$$
\tilde{\omega}(\mathbf{k})=k^{2} \tilde{\psi}(\mathbf{k}), \quad \tilde{j}(\mathbf{k})=k^{2} \tilde{a}(\mathbf{k})
$$

Terms such as $\{Z(j, a)\}_{\mathbf{k}}$ denote the Fourier transform of $Z(j, a)$, etc.

The dynamical system defined by (17) and (18) contains the quadratic nonlinear terms $\{Z(j, a)\}_{\mathbf{k}},\{Z(\psi, \omega)\}_{\mathbf{k}}$ and $\{Z(\psi, a)\}_{\mathbf{k}}$. This dynamical system is high-dimensional for $\mathcal{N}>>1$ and numerical solutions for small values of $\nu$ and $\eta$ are highly chaotic, i.e., turbulent. An efficient algorithm for numerical integration of (17) and (18) is the Fourier spectral transform method ${ }^{17}$ coupled with a third-order time-integration scheme ${ }^{21}$. The essence of this algorithm is to integrate the equations (17) and (18) in k-space and to determine the nonlinear terms such as $\{Z(j, a)\}_{\mathbf{k}}$, when needed, by Fourier transforming the separate quadratic factors back to x-space, forming products, and then transforming forward to k-space. For example, the first factor of $Z(f, g)$ appearing in $(3)$ is found from $\tilde{f}(\mathbf{k})$ using the first transform in $(12)$ :

$$
\partial_{x} f=\frac{1}{N} \sum_{\mathbf{k} \in \mathcal{K}} i k_{x} \tilde{f}(\mathbf{k}) e^{i \mathbf{k} \cdot \mathbf{x}}
$$


Once all the factors are transformed and $Z(f, g)$ is determined at each x-space grid point, we transform back to k-space:

$$
\{Z(f, g)\}_{\mathbf{k}}=\frac{1}{N} \sum_{\mathbf{x} \in \mathcal{P}} Z(f, g) e^{-i \mathbf{k} \cdot \mathbf{x}} .
$$

Note that when the transform (21) is performed, all coefficients $\{Z(f, g)\}_{\mathbf{k}}$ outside the range $1 \leq k \leq K$ are immediately set to zero (where $K=\sqrt{2} N / 3$ ). The transforms (20) and (21) are done using fast Fourier transform (FFT) routines for speed, and the whole procedure is actually done twice, once to the $\mathrm{x}$-space grid and once to a shifted $\mathrm{x}$-space grid, with the results being averaged to remove any remaining aliasing error ${ }^{20}$. This Fourier spectral transform method thereby confines the model dynamical system to the finite set of Fourier coefficients $\tilde{\omega}(\mathbf{k})$ and $\tilde{a}(\mathbf{k})$ for which $1 \leq k \leq K$. The algorithm would produce exact solutions except for time-integration and round-off errors that introduce random, quasithermal fluctuations into the model dynamical system.

These fluctuations, in fact, serve as a 'heat bath' so that the statistical mechanics of our computer model can be described in terms of canonical ensembles. In the next section we discuss the statistical theory of ideal 2-D MHD turbulence. Following this, we present numerical examples and then elucidate the origin of broken ergodicity in the dynamical system (17) and (18) that models 2-D MHD turbulence. Finally, we discuss our results and conclude this paper.

\section{STATISTICAL MECHANICS}

Statistical studies of MHD turbulence represented by Fourier modes were initiated by T. D. Lee in an early paper $^{22}$ that demonstrated the existence of canonical ensembles based on ideal invariants, as well as a Liouville theorem. The possible constants of the motion for

2-D MHD are $\widehat{E}, \widehat{H_{C}}$ and $\widehat{A}$, as defined in (5), (6) and (7), respectively. The term ideal invariants' will be used for the quantities $E=N^{2} \widehat{E}, H_{C}=N^{2} \widehat{H_{C}}$ and $A=N^{2} \widehat{A}$; using (16), these take the forms

$$
\begin{aligned}
E & =\sum_{\mathbf{k} \in \mathcal{K}^{\prime}} E(\mathbf{k}), \quad E(\mathbf{k})=k^{-2}|\tilde{\omega}(\mathbf{k})|^{2}+k^{2}|\tilde{a}(\mathbf{k})|^{2}, \\
H_{C} & =\sum_{\mathbf{k} \in \mathcal{K}^{\prime}} H_{C}(\mathbf{k}), \quad H_{C}(\mathbf{k})=\tilde{\omega}_{R}(\mathbf{k}, t) \tilde{a}_{R}(\mathbf{k})+\tilde{\omega}_{I}(\mathbf{k}, t) \tilde{a}_{I}(\mathbf{k}),
\end{aligned}
$$




$$
A=\sum_{\mathbf{k} \in \mathcal{K}^{\prime}} A(\mathbf{k}), \quad A(\mathbf{k})=|\tilde{a}(\mathbf{k})|^{2}
$$

Here, the summations are over the set of $\mathbf{k}$ defined in (14), and $E, H_{C}$ and $A$ are the total, rather than the average energy, cross helicity and squared magnetic potential; similarly, total enstrophy is $\Omega=N^{2} \widehat{\Omega}$ and total squared current is $J=N^{2} \widehat{J}$. The quantities $E, H_{C}$ and $A$ are used in the 'absolute equilibrium ensemble theory' of 2-D MHD'

This statistical mechanics of ideal 2-D MHD turbulence is based on a Gaussian canonical probability density function $(\mathrm{PDF}), D$, which can be represented as

$$
\begin{aligned}
D & =\frac{1}{Z} \exp \left(-\alpha E-\beta H_{C}-\gamma A\right) \\
Z & =\int_{\Gamma} \exp \left(-\alpha E-\beta H_{C}-\gamma A\right) d \Gamma \\
d \Gamma & =\prod_{\mathbf{k} \in \mathcal{K}^{\prime}} d \tilde{\omega}_{R}(\mathbf{k}) d \tilde{\omega}_{I}(\mathbf{k}) d \tilde{a}_{R}(\mathbf{k}) d \tilde{a}_{I}(\mathbf{k}) .
\end{aligned}
$$

In the equations above, $Z$ is the total partition function and $d \Gamma$ is the phase space volume element defined by the independent variables contained in the Fourier model (i.e., $\mathbf{k} \in \mathcal{K}^{\prime}$ ); each independent variable is integrated from $-\infty$ to $+\infty$. The set $\mathcal{K}^{\prime}$ contains $\mathcal{N}^{\prime} \cong \frac{1}{2} \pi K^{2}$ wave vectors $\mathbf{k}$ that satisfy $1 \leq k \leq K$, and the associated independent variables are $\tilde{\omega}_{R}(\mathbf{k})$, $\tilde{\omega}_{I}(\mathbf{k}), \tilde{a}_{R}(\mathbf{k})$ and $\tilde{a}_{I}(\mathbf{k})$, for a total of $N_{\Gamma}=4 \mathcal{N}^{\prime} \cong 2 \pi N^{2}$ coordinates in the phase space $\Gamma$. The PDF also applies to the case where $\mathbf{B}_{\mathrm{o}} \neq 0$, if we set $\gamma=0$ in (25) and (26).

The PDF (25) can be used to produce ensemble predictions $\langle\Phi\rangle$ of phase functions $\Phi$, which may be compared with time-averages $\bar{\Phi}$ :

$$
\langle\Phi\rangle=\int_{\Gamma} \Phi D d \Gamma, \quad \bar{\Phi}=\frac{1}{T} \int_{0}^{T} \Phi d t .
$$

The phase functions $\Phi$ in (28) are moments or sums of moments of these $N_{\Gamma}$ phase variables. Ergodicity is informally defined as occurring when $\langle\Phi\rangle=\bar{\Phi}$ for all $\Phi$, and non-ergodicity as occurring when $\langle\Phi\rangle \neq \bar{\Phi}$ for some $\Phi$.

The quantities $D$ and $Z$ in (25) and (26) can be expressed in terms of the modal quantities $\tilde{\omega}(\mathbf{k})$ and $\tilde{a}(\mathbf{k})$ as follows:

$$
D=\prod_{\mathbf{k} \in \mathcal{K}^{\prime}} D(\mathbf{k}), \quad D(\mathbf{k})=\frac{\exp [-Q(\mathbf{k})]}{Z(\mathbf{k})},
$$




$$
\begin{gathered}
Z=\prod_{\mathbf{k} \in \mathcal{K}^{\prime}} Z(\mathbf{k}), \quad Z(\mathbf{k})=\int \exp [-Q(\mathbf{k})] d \Gamma(\mathbf{k}), \\
d \Gamma(\mathbf{k})=d \tilde{\omega}_{R}(\mathbf{k}) d \tilde{\omega}_{I}(\mathbf{k}) d \tilde{a}_{R}(\mathbf{k}) d \tilde{a}_{I}(\mathbf{k}) .
\end{gathered}
$$

Again, the product in (29) is only over independent $\mathbf{k}$ and all integrations in (30) are from $-\infty$ to $+\infty$. The series (22), (23) and (24) are used in (25), and thence (29), to produce $Q(\mathbf{k})$, a necessarily positive definite, real quadratic form

$$
Q(\mathbf{k})=\alpha E(\mathbf{k})+\beta H_{C}(\mathbf{k})+\gamma A(\mathbf{k})
$$

where the expressions for $E(\mathbf{k}), H_{C}(\mathbf{k})$ and $A(\mathbf{k})$ defined in (22), (23) and (24) can be placed into $Q(\mathbf{k})$, and the modal PDF (30) can then be expressed in terms of $\tilde{\omega}(\mathbf{k})$ and $\tilde{a}(\mathbf{k})$ as

$$
D(\mathbf{k})=Z^{-1}(\mathbf{k}) \exp \left[-\tilde{y}^{\dagger}(\mathbf{k}) M_{k} \tilde{y}(\mathbf{k})\right]
$$

Here, $y^{\dagger}(\mathbf{k})$ is the Hermitian adjoint of the column vector $\tilde{y}(\mathbf{k})$, where

$$
\tilde{y}(\mathbf{k})=\left[\begin{array}{c}
\tilde{y}_{1}(\mathbf{k}) \\
\tilde{y}_{2}(\mathbf{k})
\end{array}\right]=\left[\begin{array}{c}
\tilde{\omega}(\mathbf{k}) / k \\
k \tilde{a}(\mathbf{k})
\end{array}\right] .
$$

The real, symmetric (i.e., Hermitian) $2 \times 2$ covariance matrix $M_{k}$ is

$$
M_{k}=\left[\begin{array}{cc}
\alpha & \beta / 2 \\
\beta / 2 & \alpha+\gamma / k^{2}
\end{array}\right]
$$

Now, our next step is to find the eigenvalues and eigenvectors of $M_{k}$.

\section{A. Eigenanalysis}

Diagonalization of the modal matrices $M_{k}$ in (35) is straightforward and proceeds via a similarity transformation using a unitary matrix $T_{k}$, where

$$
T_{k}=\frac{1}{\sqrt{2 C_{k} G_{k}}}\left[\begin{array}{cc}
G_{k} & \beta \\
-\beta & G_{k}
\end{array}\right], \quad C_{k}=\sqrt{\beta^{2}+\frac{\gamma^{2}}{k^{4}}}, \quad G_{k}=C_{k}-\frac{\gamma}{k^{2}} .
$$

Application of $T_{k}$ yields the diagonal matrix $\Lambda_{k}$ :

$$
\Lambda_{k}=T_{k} M_{k} T_{k}^{\dagger}=\left[\begin{array}{cc}
\lambda_{k}^{(1)} & 0 \\
0 & \lambda_{k}^{(2)}
\end{array}\right]=\left[\begin{array}{cc}
\alpha+\frac{1}{2}\left(\frac{\gamma}{k^{2}}+C_{k}\right) & 0 \\
0 & \alpha+\frac{1}{2}\left(\frac{\gamma}{k^{2}}-C_{k}\right)
\end{array}\right]
$$


Here, $T_{k}^{\dagger}$ is the Hermitian adjoint of $T_{k}$, and $\lambda_{k}^{(1,2)}$ are the eigenvalues of $M_{k}$. Although $T_{k}$ is really orthogonal and $T_{k}^{\dagger}$ is simply its transpose, the vectors $\tilde{y}(\mathbf{k})$ and eigenvectors $\tilde{v}(\mathbf{k})$ are complex, we can class $T_{k}$ as unitary. The eigenvectors $\tilde{v}(\mathbf{k})$ are

$$
\tilde{v}(\mathbf{k})=T_{k} \tilde{y}(\mathbf{k})=\left[\begin{array}{c}
\tilde{v}^{(1)}(\mathbf{k}) \\
\tilde{v}^{(2)}(\mathbf{k})
\end{array}\right]=\frac{1}{\sqrt{2 C_{k} G_{k}}}\left[\begin{array}{c}
G_{k} \tilde{\omega}(\mathbf{k}) / k+\beta k \tilde{a}(\mathbf{k}) \\
-\beta \tilde{\omega}(\mathbf{k}) / k+G_{k} k \tilde{a}(\mathbf{k})
\end{array}\right] .
$$

The complex quantities $\tilde{v}^{(1,2)}(\mathbf{k})$ will be called eigenvariables. Note that if $\mathbf{B}_{\mathrm{o}} \neq 0$, then $\gamma A=0$ in $(25)$ and the $\tilde{v}^{(1,2)}(\mathbf{k})$ become the Elsässer variables ${ }^{23}, z^{ \pm}(\mathbf{k})$, in 2-D MHD:

$$
\begin{aligned}
\lim _{\gamma A \rightarrow 0} \tilde{v}^{(1,2)}(\mathbf{k}) & = \pm \frac{1}{\sqrt{2}}\left[\frac{\tilde{\omega}(\mathbf{k})}{k} \pm k \tilde{a}(\mathbf{k})\right]= \pm \frac{1}{\sqrt{2}} z^{ \pm}(\mathbf{k}), \quad \beta>0 \\
& =\frac{1}{\sqrt{2}}\left[\frac{\tilde{\omega}(\mathbf{k})}{k} \mp k \tilde{a}(\mathbf{k})\right]=\frac{1}{\sqrt{2}} z^{\mp}(\mathbf{k}), \quad \beta<0 .
\end{aligned}
$$

Thus, the eigenvariables $\tilde{v}^{(1,2)}(\mathbf{k})$ encompass the $z^{ \pm}(\mathbf{k})$ as a special case when $\mathbf{B}_{\mathrm{o}} \neq 0$.

In terms of the real and imaginary components of the eigenvariables, $\tilde{v}_{R}^{(1,2)}(\mathbf{k})$ and $\tilde{v}_{I}^{(1,2)}(\mathbf{k})$, the modal PDF (30) takes the form

$$
\begin{aligned}
D(\mathbf{k}) & =D^{(1)}(\mathbf{k}) D^{(2)}(\mathbf{k}), \quad D^{(1,2)}(\mathbf{k})=D_{R}^{(1,2)}(\mathbf{k}) D_{I}^{(1,2)}(\mathbf{k}), \\
Z(\mathbf{k}) & =Z^{(1)}(\mathbf{k}) Z^{(2)}(\mathbf{k}), \quad Z^{(1,2)}(\mathbf{k})=Z_{R}^{(1,2)}(\mathbf{k}) Z_{I}^{(1,2)}(\mathbf{k}), \\
D_{S}^{(1,2)}(\mathbf{k}) & =\frac{\exp \left(-\lambda_{k}^{(1,2)}\left[\tilde{v}_{S}^{(1,2)}(\mathbf{k})\right]^{2}\right)}{Z_{S}^{(1,2)}(\mathbf{k})}, \quad S=R, I, \\
Z_{S}^{(1,2)}(\mathbf{k}) & =\int_{-\infty}^{\infty} \exp \left(-\lambda_{k}^{(1,2)}\left[\tilde{v}_{S}^{(1,2)}(\mathbf{k})\right]^{2}\right) d \tilde{v}_{S}^{(1,2)}(\mathbf{k})=\sqrt{\frac{\pi}{\lambda_{k}^{(1,2)}}}
\end{aligned}
$$

We can use these results to find expectation values of the modal eigenvariables, as well as expectation values for modal and total $E, H_{C}, A$, and other quantities, as needed. These expectation values will be rational functions of the 'inverse temperatures' $\alpha, \beta$ and $\gamma$, which are, at this point, undetermined. This, in turn, will lead to a relationship between $\alpha, \beta$, $\gamma$ and $E, H_{C}, A$, a relationship that will be parameterized by a single real variable. A minimization procedure will then be introduced, one that gives a unique value to the single real variable and thereby determines $\alpha, \beta$ and $\gamma$, as well as the various modal expectation 
values. Following this, we can compare these canonical ensemble predictions with timeaverages from numerical simulations. There will be a disparity between the two, a broken ergodicity that can be completely understood using eigenvalues and eigenvariables.

\section{B. Expectation Values}

Now that the total PDF (25) has been expressed as the product of the PDFs (42) of the real and imaginary components of the eigenvariables (which provide coordinates for the phase space $\Gamma$ ), we can easily determine expectation values for moments of these eigenvariables. Using (42) and (43), it is easily shown that

$$
\begin{gathered}
\left\langle\tilde{v}_{S}^{(1,2)}(\mathbf{k})\right\rangle=0, \quad\left\langle\tilde{v}_{R}^{(1,2)}(\mathbf{k}) \tilde{v}_{I}^{(1,2)}(\mathbf{k})\right\rangle=0, \\
\left\langle\left[\tilde{v}_{S}^{(1,2)}(\mathbf{k})\right]^{2}\right\rangle=\frac{1}{2 \lambda_{k}^{(1,2)}}, \quad S=R, I .
\end{gathered}
$$

Using (22), (23), (24), (38), (44) and (45), we find

$$
\begin{aligned}
\langle E(\mathbf{k})\rangle= & \left\langle\left|\tilde{v}^{(1)}(\mathbf{k})\right|^{2}\right\rangle+\left\langle\left|\tilde{v}^{(2)}(\mathbf{k})\right|^{2}\right\rangle=\frac{1}{\lambda_{k}^{(1)}}+\frac{1}{\lambda_{k}^{(2)}}=\frac{2 \alpha+\gamma / k^{2}}{\delta_{k}^{2}} \\
\left\langle H_{C}(\mathbf{k})\right\rangle= & \frac{\beta}{2 C_{k}}\left[\left\langle\left|\tilde{v}^{(1)}(\mathbf{k})\right|^{2}\right\rangle-\left\langle\left|\tilde{v}^{(2)}(\mathbf{k})\right|^{2}\right\rangle\right] \\
= & \frac{\beta}{2 C_{k}}\left[\frac{1}{\lambda_{k}^{(1)}}-\frac{1}{\lambda_{k}^{(2)}}\right]=-\frac{\beta}{2 \delta_{k}^{2}}, \\
\langle A(\mathbf{k})\rangle= & \frac{C_{k}-\gamma / k^{2}}{2 k^{2} C_{k}}\left\langle\left|\tilde{v}^{(1)}(\mathbf{k})\right|^{2}\right\rangle+\frac{C_{k}+\gamma / k^{2}}{2 k^{2} C_{k}}\left\langle\left|\tilde{v}^{(2)}(\mathbf{k})\right|^{2}\right\rangle \\
= & \frac{C_{k}-\gamma / k^{2}}{2 k^{2} C_{k} \lambda_{k}^{(1)}}+\frac{C_{k}+\gamma / k^{2}}{2 k^{2} C_{k} \lambda_{k}^{(2)}}=\frac{\alpha}{k^{2} \delta_{k}^{2}}, \\
& \delta_{k}^{2} \equiv \alpha^{2}-\beta^{2} / 4+\alpha \gamma / k^{2} .
\end{aligned}
$$

In summary, we have

$$
\langle E(\mathbf{k})\rangle=\frac{2 \alpha+\gamma / k^{2}}{\delta_{k}^{2}}, \quad\left\langle H_{C}(\mathbf{k})\right\rangle=-\frac{\beta}{2 \delta_{k}^{2}}, \quad\langle A(\mathbf{k})\rangle=\frac{\alpha}{k^{2} \delta_{k}^{2}} .
$$


These are individual mode contributions to the expectation values of the ideal invariants. Note that, since $H_{C}$ is a pseudoscalar under the parity transformation $\mathbf{x} \rightarrow-\mathbf{x}$, so is $\beta$.

We can also find the modal expectation value of quantities that are not ideal invariants, such as the magnetic energy; here $\delta_{k}^{2}$ is given in (49):

$$
\left\langle E_{M}(\mathbf{k})\right\rangle=k^{2}\langle A(\mathbf{k})\rangle=\frac{\alpha}{\delta_{k}^{2}}
$$

Note that $\lambda_{k}^{(1,2)}>0 \rightarrow \lambda_{k}^{(1)} \lambda_{k}^{(2)}=\delta_{k}^{2}>0$, and since $\langle A(\mathbf{k})\rangle>0$, we have $\alpha>0$. However, (50) tells us that $\beta\left\langle H_{C}(\mathbf{k})\right\rangle<0$, so $\beta$ takes a sign opposite to that of $\left\langle H_{C}(\mathbf{k})\right\rangle$. Also, since $\langle E(\mathbf{k})\rangle>0$ for all $k$, then $\gamma>-2 \alpha$, i.e., $\gamma$ can be positive, zero or negative.

Now that we have various modal expectation values given in terms of $\alpha, \beta$ and $\gamma$, we can sum these and invert the results to find the heretofore unknown $\alpha, \beta$ and $\gamma$ in terms of the three integral invariants and one unknown parameter. We then use a minimization procedure to determine this unknown parameter and thereby determine $\alpha, \beta$ and $\gamma$, allowing us to assign values to ensemble predictions and to compare these to time averages found through numerical simulation. This, in turn, leads us to the mechanism of broken ergodicity.

\section{Inverse Temperatures}

Using (50) and (51), it is easy to verify that

$$
\begin{aligned}
& \alpha\langle E(\mathbf{k})\rangle+\beta\left\langle H_{C}(\mathbf{k})\right\rangle+\gamma\langle A(\mathbf{k})\rangle=2, \\
& \alpha\langle E(\mathbf{k})\rangle+\frac{4 \alpha^{2}}{\beta}\left\langle H_{C}(\mathbf{k})\right\rangle-\gamma\langle A(\mathbf{k})\rangle=0, \\
& 2 \alpha\left\langle H_{C}(\mathbf{k})\right\rangle+\beta\left\langle E_{M}(\mathbf{k})\right\rangle=0 .
\end{aligned}
$$

In what follows, we can set the expectation of values of the ideal invariants equal to their initial values, $\mathcal{E}, \mathcal{H}_{C}$ and $\mathcal{A}$, since these are expected to be constant, and set the expectation value of the magnetic energy equal to the variable $\varphi$, which is initially unknown, i.e.,

$$
\langle E\rangle=\mathcal{E}, \quad\left\langle H_{C}\right\rangle=\mathcal{H}_{C}, \quad\langle A\rangle=\mathcal{A}, \quad\left\langle E_{M}\right\rangle=\varphi
$$

Now, we sum (52), (53) and (54) over the $\mathcal{N}^{\prime}$ wavevectors $\mathbf{k} \in \mathcal{K}^{\prime}$, using (55), to get

$$
\alpha \mathcal{E}+\beta \mathcal{H}_{C}+\gamma \mathcal{A}=2 \mathcal{N}^{\prime}, \quad \alpha \mathcal{E}+4 \frac{\alpha^{2}}{\beta} \mathcal{H}_{C}-\gamma \mathcal{A}=0, \quad 2 \alpha \mathcal{H}_{C}+\beta \varphi=0 .
$$


The equations (56) can be readily solved to yield the inverse temperatures in terms of the quantities $\mathcal{E}, \mathcal{H}_{C}, \mathcal{A}$ and $\varphi$; in terms of the grid-averaged values, e.g., $\widehat{\varphi}=\varphi / N^{2}$, we have

$$
\alpha=\frac{n \widehat{\varphi}}{\widehat{\varphi}(\widehat{\mathcal{E}}-\widehat{\varphi})-\widehat{\mathcal{H}}_{C}^{2}}, \quad \beta=-2 \frac{\widehat{\mathcal{H}}}{\widehat{\varphi}} \alpha, \quad \gamma=\frac{\widehat{\mathcal{E}}-2 \widehat{\varphi}}{\widehat{\mathcal{A}}} \alpha .
$$

Here, $n=\mathcal{N}^{\prime} / N^{2}$, where $\mathcal{N}^{\prime} \cong \pi K^{2} / 2$ and $K=\sqrt{2} N / 3$, so that $n \cong \pi / 9 \cong 0.3491$. Thus, the undetermined multipliers $\alpha, \beta$ and $\gamma$ found in the PDF (25) are, in fact, all functions of one initially unknown variable $\widehat{\varphi}$. Now, $|\widehat{\mathcal{H}}|<\widehat{\mathcal{E}}$ and $0<\widehat{\mathcal{A}} \leq \widehat{\varphi}<1$, and if $\widehat{\mathcal{E}}=1$, then $\alpha$, $\beta$ and $\gamma$ are all roughly of order unity, unless the denominators in the expressions are close to zero, which may occur.

\section{The Entropy Functional}

The parameters $\alpha, \beta$ and $\gamma$ can be determined from the constant values $\widehat{\mathcal{E}}, \widehat{\mathcal{H}_{C}}$ and $\widehat{\mathcal{A}}$, as described by the relations (57), by minimizing an entropy functional ${ }^{24,25}$ with respect to $\widehat{\varphi}$. Here, we minimize the specific entropy functional $\sigma(\widehat{\varphi})$, using the various formulae given above,

$$
\begin{aligned}
\sigma(\widehat{\varphi}) & =-\frac{1}{N^{2}}\langle\ln D\rangle=2 n(1+\ln \pi)-\frac{1}{N^{2}} \sum_{\mathbf{k} \in \mathcal{K}^{\prime}} \ln \delta_{k}^{2} \\
\delta_{k}^{2} & =\alpha^{2}\left(1-\frac{\widehat{\mathcal{H}}_{C}^{2}}{\widehat{\varphi}^{2}}+\frac{\widehat{\mathcal{E}}-2 \widehat{\varphi}}{\widehat{\mathcal{A}} k^{2}}\right) .
\end{aligned}
$$

The form of $\delta_{k}^{2}$ comes from using (49), along with the $\alpha(\widehat{\varphi}), \beta(\widehat{\varphi}), \gamma(\widehat{\varphi})$ and $n$, as defined in (57). Please note that, as discussed by Khinchin ${ }^{24}$, the entropy of a closed system, such as the finite Fourier model we are studying, is not a dynamic quantity that depends on the time-dependent value of $\varphi$ on a phase trajectory, but rather a global property of the ensemble phase space with a value of $s=\sigma\left(\widehat{\varphi}_{\mathrm{o}}\right)$ determined by finding the $\widehat{\varphi}=\widehat{\varphi}_{\mathrm{o}}$ that minimizes $\sigma(\widehat{\varphi})$. Using the fact that $\alpha>0$ and $\delta_{k}^{2}>0$, it can be shown that $-\infty<s \leq 1.7482+2 \ln \widehat{\mathcal{E}}$.

In an attempt to determine the minimum of $\sigma(\widehat{\varphi})$, we can try to find the zeroes of its first derivative with respect to $\widehat{\varphi}$. This first derivative is

$$
\frac{d \sigma(\widehat{\varphi})}{d \widehat{\varphi}}=\sigma^{\prime}(\widehat{\varphi})=F(\widehat{\varphi}) G(\widehat{\varphi})
$$




$$
\begin{aligned}
& F(\widehat{\varphi})=\frac{\widehat{\varphi}^{3}-\widehat{\mathcal{H}}_{C}^{2}(3 \widehat{\varphi}-\widehat{\mathcal{E}})}{\widehat{\varphi}^{2}\left[\widehat{\varphi}(\widehat{\mathcal{E}}-\widehat{\varphi})-\widehat{\mathcal{H}}_{C}^{2}\right]}, \\
& G(\widehat{\varphi})=\frac{2}{N^{2}} \sum_{\mathbf{k} \in \mathcal{K}^{\prime}} \frac{\hat{\mathcal{E}}-2 \widehat{\varphi}+\mathcal{A} k^{2}\left(1-\widehat{\mathcal{H}}_{C}^{2} / \widehat{\varphi}^{2}\right)}{\widehat{\widehat{\varphi}}} .
\end{aligned}
$$

Now, $\langle\mathbf{u} \cdot \mathbf{b}\rangle^{2}<\left\langle u^{2}\right\rangle\left\langle b^{2}\right\rangle$ implies $\widehat{\mathcal{H}}_{C}^{2}<\widehat{\varphi}(\widehat{\mathcal{E}}-\widehat{\varphi})$, so that the denominator of $F(\widehat{\varphi})$ must be positive. Then, $\widehat{\varphi}_{-}<\widehat{\varphi}<\widehat{\varphi}_{+}$, where $\widehat{\varphi}_{ \pm}=\frac{1}{2}\left(\widehat{\mathcal{E}} \pm \sqrt{\widehat{\mathcal{E}}^{2}-4 \widehat{\mathcal{H}}_{C}^{2}}\right)$; since $\left\langle|\mathbf{u} \pm \mathbf{b}|^{2}\right\rangle>0$ implies $2\left|\widehat{\mathcal{H}_{C}}\right|<\widehat{\mathcal{E}}$, the $\widehat{\varphi}^{ \pm}$are real. The numerator of $F(\widehat{\varphi})$ has a mimimum at $\widehat{\varphi}=\left|\widehat{\mathcal{H}_{C}}\right|=$ $\sqrt{\hat{\varphi}^{+} \widehat{\varphi}^{-}}$, at which value $F\left(\left|\widehat{\mathcal{H}_{C}}\right|\right)={\widehat{\mathcal{H}_{C}}}^{-1}>0$, so that $F(\widehat{\varphi})>0$ for acceptable values of $\widehat{\varphi}$. Thus, the zeroes of $\sigma^{\prime}(\widehat{\varphi})$ are the zeroes of $G(\widehat{\varphi})$.

Although there is no room here for an extensive discussion, there is only one value $\widehat{\varphi}=\widehat{\varphi}_{\mathrm{o}}$ such that $G\left(\widehat{\varphi}_{\mathrm{o}}\right)=0$. Finding $\hat{\varphi}_{\mathrm{o}}$ requires a numerical procedure, except when $\mathbf{B}_{\mathrm{o}} \neq 0$ (see below). Although $G^{\prime}(\widehat{\varphi})=d G / d t>0$ can alternate in sign as $\widehat{\varphi}$ is varied, we always have $G^{\prime}(\widehat{\varphi})>0$ at $\widehat{\varphi}=\widehat{\varphi}_{\mathrm{o}}$, where (after some algebraic manipulation),

$$
\begin{gathered}
G^{\prime}\left(\widehat{\varphi}_{\mathrm{o}}\right)=g^{\prime}\left(p_{\mathrm{o}}\right)=\frac{2}{N^{2}} \sum_{\mathbf{k} \in \mathcal{K}^{\prime}} \frac{1-a_{k}\left(1+3 h^{2} / p_{\mathrm{o}}^{2}\right)+2 a_{k}^{2} h^{2} / p_{\mathrm{o}}^{3}}{\left[1-2 p_{\mathrm{o}}+a_{k}\left(1-h^{2} / p_{\mathrm{o}}^{2}\right)\right]^{2}}, \\
p_{\mathrm{o}}=\widehat{\varphi}_{\mathrm{o}} / \widehat{\mathcal{E}}, \quad a_{k}=\widehat{\mathcal{A}} k^{2} / \widehat{\mathcal{E}}, \quad h=\left|\widehat{\mathcal{H}_{C}}\right| / \widehat{\mathcal{E}} .
\end{gathered}
$$

The sum above contains terms that have a quadratic function of $a_{k}$ in the numerator. It can be shown that the quadratic is positive for all values of $a_{k}$ except possibly for a limited, inconsequential range of $a_{k}$, while the corresponding denominators are always positive. The net result is that we always have $g^{\prime}\left(p_{\mathrm{o}}\right)>0$ and that $\widehat{\varphi}=\widehat{\varphi}_{\mathrm{o}}$ minimizes $\sigma(\widehat{\varphi})$, yielding the specific entropy $s=\sigma\left(\widehat{\varphi}_{\mathrm{o}}\right)$. Once $\widehat{\varphi}_{\mathrm{o}}$ is found, the inverse temperatures (57) are determined and ensemble predictions such as (50) can be assigned definite values.

\section{E. Nonzero Mean Magnetic Field}

The statistical mechanics of the case where $\mathbf{B}_{\mathrm{o}} \neq 0$ has a much simpler formulation than the $\mathbf{B}_{\mathrm{o}}=0$ case given above. In the $\mathbf{B}_{\mathrm{o}} \neq 0$ case, $\gamma=0$, or equivalently, $\widehat{\varphi}_{\mathrm{o}} \equiv \widehat{\mathcal{E}} / 2$, which leads to eigenvalues independent of $k$ :

$$
\lambda_{k}^{(1,2)}=\alpha \pm \frac{|\beta|}{2}=\frac{2 n}{\widehat{\mathcal{E}} \mp 2\left|\widehat{\mathcal{H}_{C}}\right|} .
$$


In this case, the specific entropy is determined only by $\widehat{\mathcal{H}_{C}}$ (where, $\mathrm{e}=2.718 \ldots$ and $\left.n \cong \pi / 9\right)$ :

$$
s=n \ln \left[\left(\frac{\pi \mathrm{e}}{2 n}\right)^{2}\left(\widehat{\mathcal{E}}^{2}-4 \widehat{\mathcal{H}}_{C}^{2}\right)\right] .
$$

Above, $\left|\widehat{\mathcal{H}_{C}}\right|<\mathcal{E} / 2$, since we cannot have $\left|\widehat{\mathcal{H}_{C}}\right|=\mathcal{E} / 2$ because then either $z^{+}(\mathbf{k})=0$ or $z^{-}(\mathbf{k})=0$ for all $\mathbf{k} \in \mathcal{K}^{\prime}$, all nonlinear interactions are absent, and there is no turbulence. It is clear from the form of $s$ in (65) that the maximum that $s$ can take occurs at $\widehat{\mathcal{H}_{C}}=0$, where (again) $s_{\max } \cong 1.7482+2 \ln \widehat{\mathcal{E}}$, while the minimum $s_{\min }$ is unbounded from below as $\left|\widehat{\mathcal{H}}{ }_{C}\right| \rightarrow \mathcal{E} / 2$. These limits are general for ideal 2-D MHD turbulence, as they also apply to the case where $\mathbf{B}_{\mathrm{o}}=0$, as already mentioned.

There are $n$ eigenvariables $\tilde{v}^{(1)}(\mathbf{k})$ and $n$ eigenvariables $\tilde{v}^{(2)}(\mathbf{k})$ and together they have an average energy of $E /\left(2 \mathcal{N}^{\prime}\right)=\mathcal{E} /(2 n)$. Individually, from (45), the eigenvariables have the following expectation values for their energy:

$$
\left\langle\left|\tilde{v}^{(1,2)}(\mathbf{k})\right|^{2}\right\rangle=\frac{1}{\lambda_{k}^{(1,2)}} .
$$

In the $\mathbf{B}_{\mathrm{o}} \neq 0$ and $\widehat{\mathcal{H}_{C}}=0$ case, $s=s_{\max }$ and (65) and tells us that $\lambda_{k}^{(1)}=\lambda_{k}^{(2)}=\mathcal{E} /(2 n)$, so that all eigenvariables have identical expected energies of $\widehat{\mathcal{E}} /(2 n)$. However, if $\left|\widehat{\mathcal{H}_{C}}\right| \rightarrow \mathcal{E} / 2$, then $\lambda_{k}^{(1)} \rightarrow \infty$, as seen in (64), and we have

$$
\left\langle E^{(1)}(\mathbf{k})\right\rangle \rightarrow 0, \quad\left\langle E^{(2)}(\mathbf{k})\right\rangle \rightarrow \frac{\mathcal{E}}{n} .
$$

Thus, when $\mathbf{B}_{\mathrm{o}} \neq 0$ and $\left|\widehat{\mathcal{H}_{C}}\right| \rightarrow \widehat{\mathcal{E}} / 2$, the Elsässer variables separate into one half with energies that are very large when compared to the other half. In the formalism given here, $\tilde{v}^{(2)}(\mathbf{k})$ is the half that always contains larger energies. Using (39) and (50), we see that for $\beta>0$, the $\tilde{v}^{(2)}(\mathbf{k})$ are (except for a minus sign) the Elsässer variables $z^{-}(\mathbf{k})$ corresponding to $\widehat{\mathcal{H}_{C}}<0$, while for $\beta<0$, the $\tilde{v}^{(2)}(\mathbf{k})$ are the $z^{+}(\mathbf{k})$ corresponding to $\widehat{\mathcal{H}_{C}}>0$.

\section{NUMERICAL RESULTS}

As already mentioned, we numerically simulated the dynamical system defined by (17) and (18) by using a Fourier spectral transform method ${ }^{17}$ coupled with a third-order timeintegration scheme ${ }^{21}$. Here, we present twelve ideal runs $(\nu=\eta=0)$ whose parameters are given in Table I. For all runs, $\mathbf{B}_{\mathrm{o}}=0, N=128$, the time step size was $\Delta t=10^{-3}$, and each run lasted $10^{6}$ time steps, that is, from simulation time $t=0$ to $t=1000$. 
TABLE I: Numerical Simulation Parameters.

\begin{tabular}{ccccccccc}
\hline \hline Run & $\widehat{\mathcal{E}}$ & $\widehat{\mathcal{H}_{C}}$ & $\widehat{\mathcal{A}}$ & $\alpha$ & $\beta$ & $\gamma$ & $\widehat{\varphi}_{\mathrm{o}}$ & $s$ \\
\hline $\mathrm{A}$ & 1.00007 & 0.35501 & 0.42000 & 2.2032 & -2.4189 & -1.5390 & 0.64672 & 1.0730 \\
$\mathrm{~B}$ & 1.00000 & 0.47309 & 0.18632 & 6.9125 & -12.717 & -1.0633 & 0.51433 & 0.80260 \\
$\mathrm{C}$ & 1.00006 & 0.15709 & 0.18632 & 0.93966 & -0.50343 & -0.87158 & 0.58644 & 1.5687 \\
$\mathrm{D}$ & 1.00003 & -0.034993 & 0.012160 & 0.71012 & 0.098225 & -0.69560 & 0.50597 & 1.7395 \\
$\mathrm{E}$ & 1.00000 & 0.49931 & 0.18632 & 253.49 & -505.75 & -1.1105 & 0.50041 & -0.46998 \\
$\mathrm{~F}$ & 1.00006 & -0.0069888 & 0.18632 & 0.85806 & 0.020222 & -0.85729 & 0.59310 & 1.6061 \\
$\mathrm{G}$ & 1.00005 & 0.25147 & 0.18632 & 1.1090 & -0.96946 & -0.89648 & 0.57533 & 1.5010 \\
$\mathrm{H}$ & 1.00000 & 0.47138 & 0.00031952 & 6.1330 & -11.953 & 624.80 & 0.48372 & 0.92079 \\
$\mathrm{I}$ & 1.00000 & 0.39998 & 0.00015964 & 1.8322 & -4.2739 & 3605.3 & 0.34293 & 1.0983 \\
$\mathrm{~J}$ & 1.00000 & 0.38998 & 0.00005499 & 17.250 & -69.565 & 192350 & 0.19340 & -0.87838 \\
$\mathrm{~K}$ & 1.00000 & 0.45960 & 0.000091651 & 20.207 & -58.490 & 80445. & 0.31757 & -0.62948 \\
$\mathrm{~L}$ & 1.00000 & 0.38998 & 0.00021996 & 1.5870 & -3.1358 & 1519.0 & 0.39474 & 1.2773 \\
\hline \hline
\end{tabular}

In Table I, the values (to five significant figures) given for $\widehat{\mathcal{E}}, \widehat{\mathcal{H}_{C}}$ and $\widehat{\mathcal{A}}$ are the timeaveraged values over each complete run; at the beginning of each run, $\widehat{\mathcal{E}}=1$, and the timeaveraged values differ by less than 1 part in $10^{4}$, indicating the accuracy of the numerical $\operatorname{method}\left(\widehat{\mathcal{H}_{C}}\right.$ and $\widehat{\mathcal{A}}$ were similarly well conserved). These values of $\widehat{\mathcal{E}}, \widehat{\mathcal{H}_{C}}$ and $\widehat{\mathcal{A}}$ were used in (58) to find $\alpha, \beta$ and $\gamma$ by determining the value $\widehat{\varphi}_{\mathrm{o}}$ that minimized $\sigma(\widehat{\varphi})$ to find the entropy $s=\sigma\left(\widehat{\varphi}_{\mathrm{o}}\right)$; all these values, for each run, appear in Table I.

Although $10^{6}$ time steps might seem to be sufficient for the model dynamical system to reach equilibrium, Fig. 1 indicates that this is not always the case. In this figure, the values of mean square current $\widehat{J}$ for Runs $B, C, E, F$ and $G$ (which all have $\widehat{\mathcal{A}}=0.18632$ ) are presented. While Runs C, F and G appear to have reached stationarity relatively quickly, and Run B soon thereafter, the value of $\widehat{J}$ for Run E is still increasing. In looking at Table I, we see that Run $\mathrm{E}$ has the highest value of $\widehat{\mathcal{H}_{C}}(=0.49931)$, which indicates that $\mathbf{u} \approx \mathbf{b}$, effectively bottlenecking the nonlinear transfer of energy from low to high wave numbers. In contrast, Run $\mathrm{F}$ has $\widehat{\mathcal{H}_{C}}=-0.007$ and reaches its terminal value the quickest of all.

In regard to the Runs $\mathrm{B}, \mathrm{C}, \mathrm{E}, \mathrm{F}$ and $\mathrm{G}$ (all with $\widehat{\mathcal{A}}=0.18632$ ), Table I gives their 


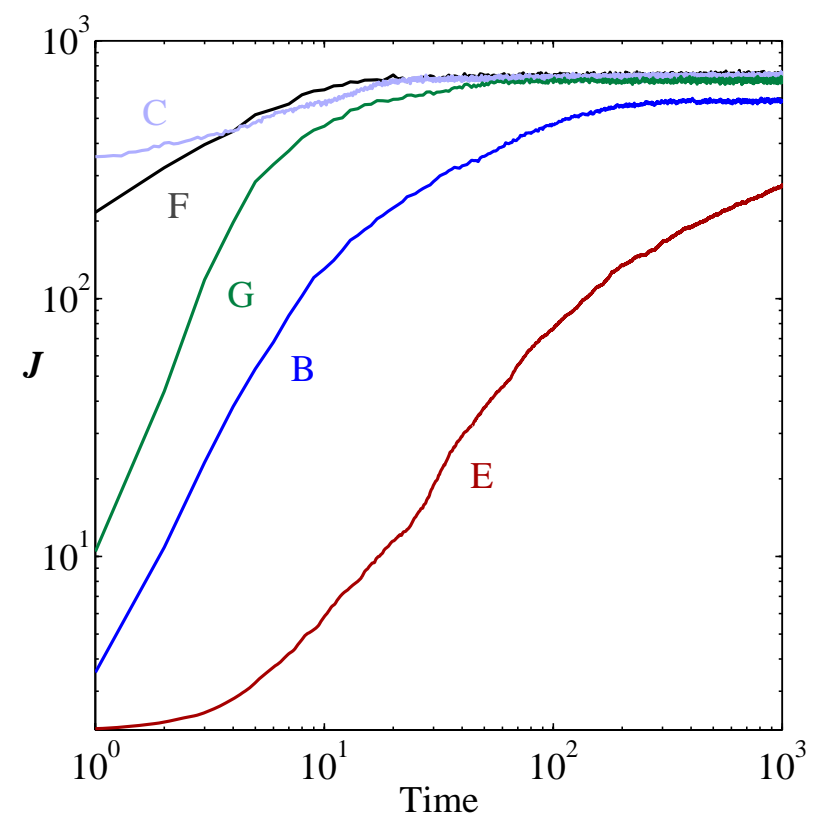

FIG. 1: Mean square current $J$ for Runs B, C, E, F and G.

respective values of $\widehat{\mathcal{H}_{C}}$; correlating these values with the behavior shown in Fig. 1 indicates that the time needed to reach the terminal value of $\widehat{J}$ is directly correlated to the magnitude of $\widehat{\mathcal{H}_{C}}$. However, for Runs H, I, J, K and L, which all have relatively high values of $\left|\widehat{\mathcal{H}_{C}}\right|$ but very low values of $\widehat{A}$, graphs analogous to Fig. 1 show these runs reached equilibrium quicker than any runs in Fig. 1. The remaining runs of Table I, Runs A and D, were similarly seen to have achieved equilibrium at about the same rate as Run G, shown in Fig. 1.

A major qualitative difference of various runs in Table $I$ is due to the value of $\widehat{\varphi}_{\mathrm{o}}$ : All runs with $\widehat{\varphi}_{\mathrm{o}}>\frac{1}{2}(\gamma<0)$, such as Run A, peak at $k=1$, while runs with $\widehat{\varphi}_{\mathrm{o}}<\frac{1}{2}(\gamma>0)$, such as Run J, peak at $k=K$, the largest wavenumber in the simulation. The total ideal energy spectra for the eigenmodes can be calculated from the eigenvalues (37) and is $E^{(1,2)}(k)=\pi k / \lambda_{k}^{(1,2)}$; example spectra for Runs A and J are shown in Fig. 2. Run A $\left(\widehat{\varphi}_{\mathrm{o}}>\frac{1}{2}\right.$, $\gamma<0)$ peaks at lowest $k$ and Run $\mathrm{J}\left(\widehat{\varphi}_{\mathrm{o}}<\frac{1}{2}, \gamma>0\right)$ peaks at largest $k$; if $\widehat{\varphi}_{\mathrm{o}}=\frac{1}{2}$ exactly $(\gamma \equiv 0)$, as is the case when $\mathbf{B}_{\mathrm{o}} \neq 0$, the predicted spectra are precisely flat; if $\widehat{\varphi}_{\mathrm{o}} \simeq \frac{1}{2}$, as in Run E, then the expectation may be that the $E^{(1,2)}(k)$ will be relatively flat, but it is not. Instead, the spectra for Run E are very similar to the spectra for Run A, as shown in Fig. 2. Let us now take a more detailed look at the energy spectra for some of these runs.

Two-dimensional spectra can be generated by determining the time-averages and vari- 


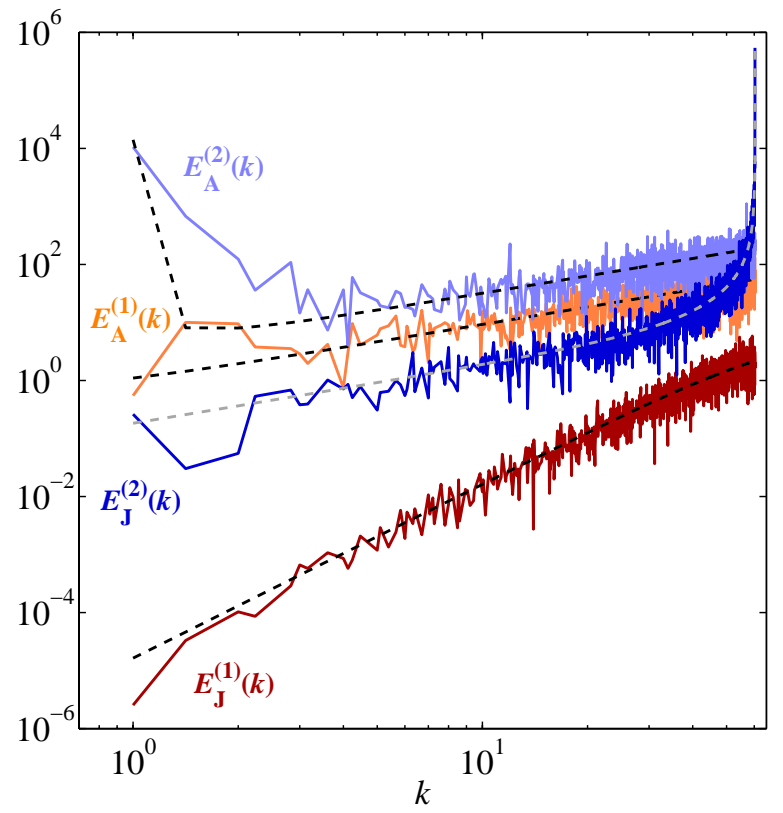

FIG. 2: Ideal energy spectra for Runs A and J.

ances, over $t=0$ to 1000, for all of the Fourier coefficients of any given run. Average values and variances with respect to time will be denoted by $v_{A}^{(s)}(\mathbf{k})$ and $\left|v^{(s)}(\mathbf{k})\right|_{V}^{2}(s=1,2)$, respectively; these are used to define the two-dimensional energy spectra

$$
E_{A}(\mathbf{k})=\sum_{s=1}^{2}\left|v_{A}^{(s)}(\mathbf{k})\right|^{2}, \quad E_{V}(\mathbf{k})=\sum_{s=1}^{2}\left|v^{(s)}(\mathbf{k})\right|_{V}^{2}, \quad E_{T}(\mathbf{k})=E_{A}(\mathbf{k})+E_{V}(\mathbf{k}) .
$$

These spectra are shown in Fig. 3 for Run E and are representative of the $\widehat{\varphi}_{\mathrm{o}}>\frac{1}{2}$ runs in Table I. Runs with $\widehat{\varphi}_{\mathrm{o}}>\frac{1}{2}$ (such as Run J in Fig. 2) peak at $k=K$ but have a similar relation between mean, variance and total two-dimensional spectra.

The critical feature appearing in Fig. 3 is that $E_{A}(\mathbf{k})$ is not close to zero everywhere, but instead is relatively large at low $k$. In fact, at low $k$ it gives the principal contribution to $E_{T}(\mathbf{k})$. The statistical theory does not predict this phenomenon and something obviously is occurring that 'breaks the ergodicity' of the dynamical system under consideration.

Returning to Fig. 2, what we see, for Runs $\mathrm{A}$ and $\mathrm{J}$, is a comparison of the computed values (at $t=1000$ ) of $E^{(1,2)}(k)=\pi k\left|v^{(1,2)}(\mathbf{k})\right|_{\text {avg }}^{2}$ (here, 'avg' signifies values with the same $k$ are averaged at a specific time $t$ ) with expected values (the dashed curves) determined by (66). The fit seems fairly good, particularly as $k$ increases, and is representative of the level of closeness between predicted and computed values of $E^{(1,2)}(k)$ for the runs in Table I. However, at low $k$, there appears to be less of a match than as $k \rightarrow K$, and Fig. 3 indicates 

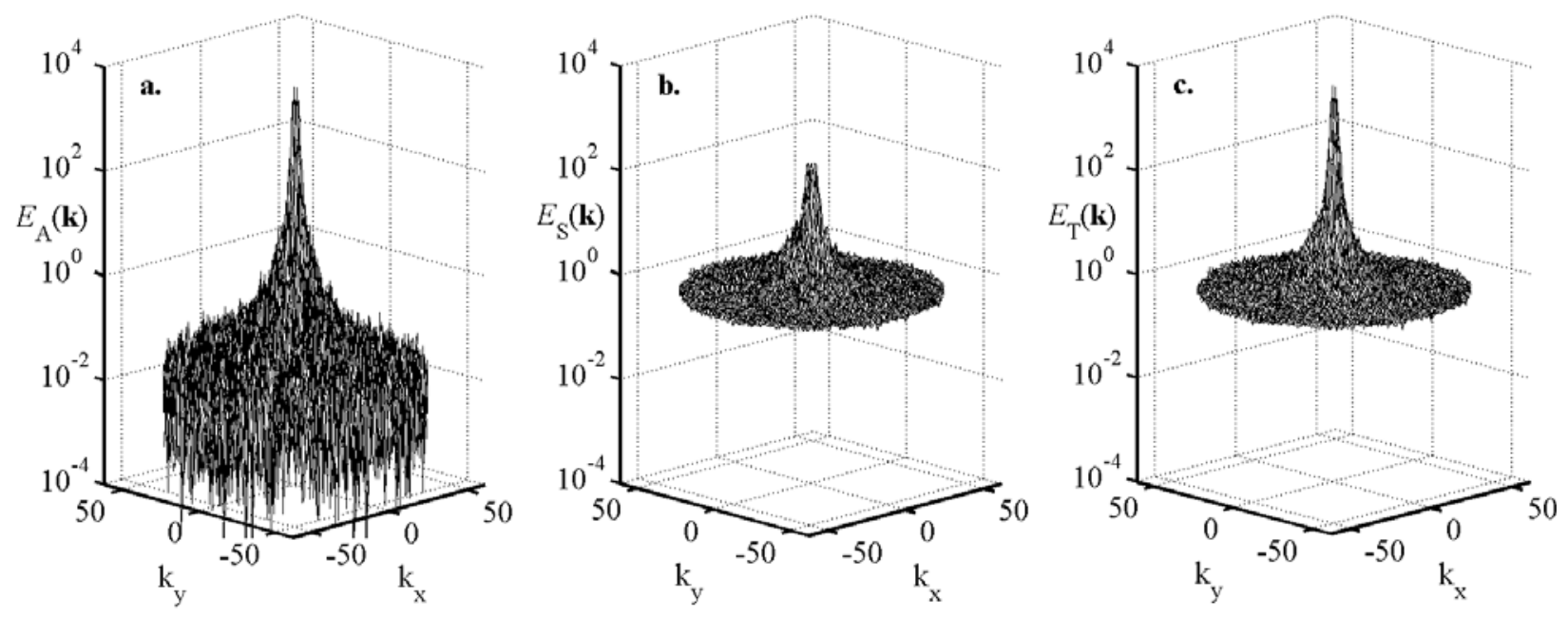

FIG. 3: Run E, 2-D spectra. Energy in a. time-averages of the Fourier coefficients; b. variances (with respect to a.); c. total (sum of a. plus b.).

that these unexpected values are not, in fact, momentary temporal fluctuation. We consider this further by now discussing the behavior if individual eigenmodes.

In addition to recording instantaneous spectra, such as in Fig. 2, or examining timestatistics of spectra, as in Fig. 3, we also followed the time evolution of certain $v^{(s)}(\mathbf{k})$ $(s=1,2)$ for selected values of $k$. These can be used to produce time histories of $E^{(1,2)}(k)$, as well as phase trajectories, for these selected $k$. In Fig. 4 , we present $E^{(1,2)}(k)$ vs time for $k=1,2$ and 4, from Run A; in this figure, we also have expected values for each of the $E^{(1,2)}(k)$. What we see is that, although the computed and expected $E^{(1,2)}(k)$ for $k=1$ fall on top of one another, those for $k=2$ and 4 do not. Instead, the simulation values are anomalously high, commensurate with what we see in Fig. 2 at low $k$. Thus, although computed spectra appear much as predicted over moderate to large $k$, this is another clear indication that some form of non-ergodicity is present.

In Figs. 5 and 6 , both pertaining to Run A, we see more detail concerning the time histories shown in Fig. 4. These figures are 'phase trajectories', i.e., projections of the path the dynamical system is following in phase space onto a two-dimensional plane whose abscissa and ordinate are the real part and imaginary parts of a particular eigenvariable $v^{(1,2)}(\mathbf{k})$. Although Fig. 4 indicates that computed and expected values $E^{(2)}(k)$ for $k=1$ match, when we look the behavior of the individual coefficients in Fig. 5, we see that the mean magnitude of $v^{(2)}(\mathbf{k}), \mathbf{k}=(0,1)$ is significantly larger than expected (86.1 vs 66.9), 


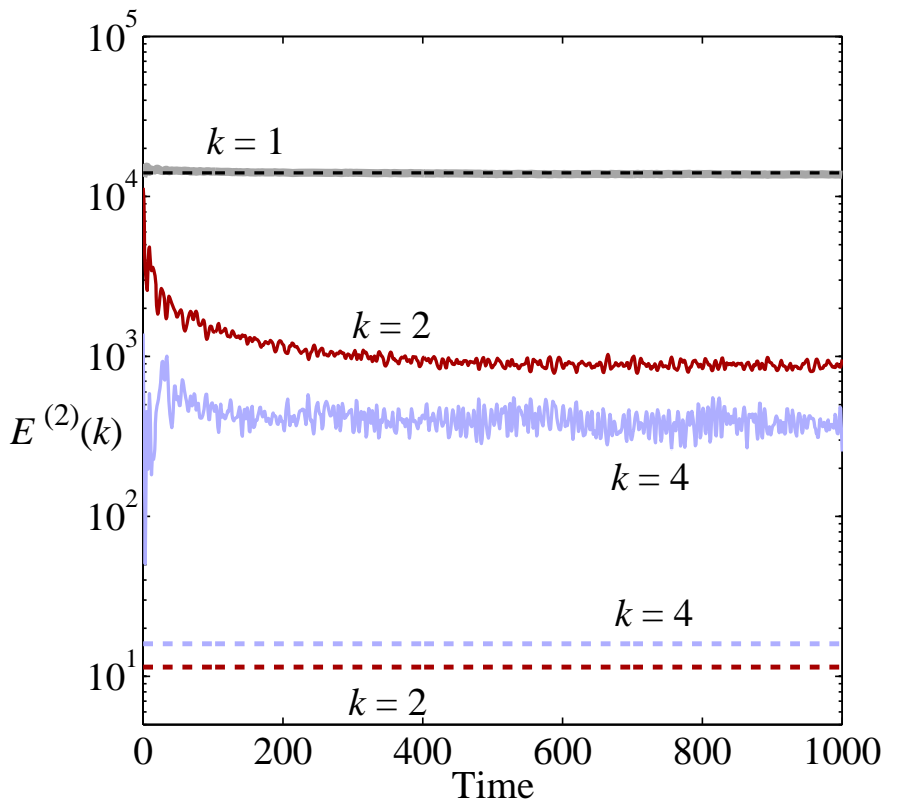

FIG. 4: Time histories of $E^{(s)}(k), s=1,2$, for $k=1,2$ and 4, from Run A.

while the mean magnitude of $v^{(2)}(\mathbf{k}), \mathbf{k}=(1,0)$ is significantly smaller than expected (37.0 vs 66.9$)$; the coefficients $v^{(1)}(\mathbf{k})$ have close to the expected magnitudes $(\approx 0.59)$. Also, note that $v^{(2)}(\mathbf{k}), \mathbf{k}=(1,0)$ behaves like an Alfvén wave, propagating on the almost stationary magnetic field associated with $v^{(2)}(\mathbf{k}), \mathbf{k}=(0,1)$.

In Fig. 6, we have phase trajectories for $v^{(1,2)}(\mathbf{k}), k=2$ and 4 , of Run A. In this figure, the annular phase trajectories (of the coefficients with $k_{x} \neq 0$ ) also represent Alfvén waves on $v^{(2)}(0,1)$. The mean magnitudes of $v^{(2)}(\mathbf{k}), \mathbf{k}=(1,1)$ and $(-1,1)$, are significantly larger than expected (11.9 vs 1.35 ), and the mean magnitude of $v^{(1)}(1,1)$ is also significantly larger than expected (1.74 vs 0.57 ). Turning to the $k^{2}=4$ coefficients, he mean magnitudes of $v^{(2)}(\mathbf{k})$, $\mathbf{k}=(0,2)$ and $(2,0)$, are also significantly larger than expected (5.39 and 5.78, respectively, vs 1.35$)$, while the mean magnitude of $v^{(1)}(0,2)$ is also significantly larger than expected $(1.93$ vs 0.56$)$.

An example of more ordinary behavior (which absolute equilibrium ensemble theory expects to apply all coefficients) is seen in Fig. 7, where the phase trajectory of $v^{(1)}(\mathbf{k})$, for $\mathbf{k}=(0,1)$ from Run $\mathrm{D}$ is given. The mean seems to be around zero and the standard deviation of 1.22 appears to be about the predicted value of 1.18. However, if we look at $v^{(2)}(\mathbf{k}), k=1$, anomalous behavior is again observed. In Fig. 8, we see phase trajectories for 


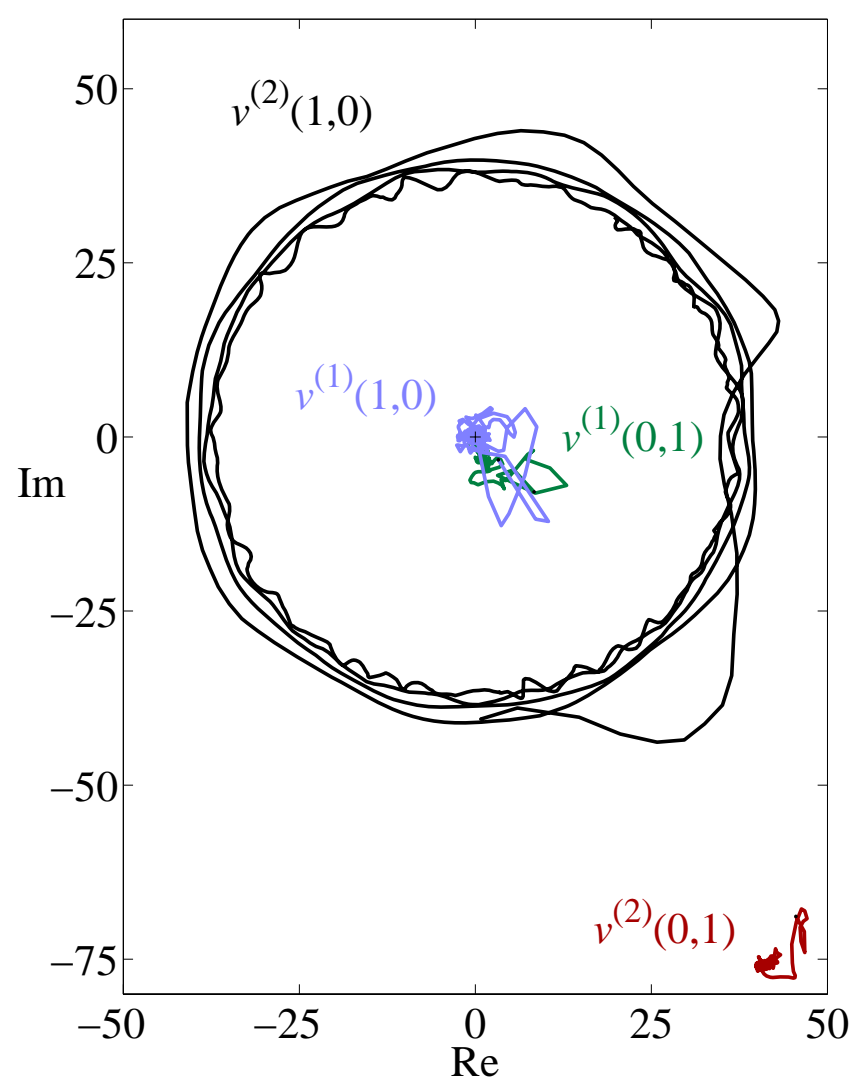

FIG. 5: Phase trajectories of $v^{(1,2)}(\mathbf{k})$ with $k^{2}=1$, for Run A.

$v^{(2)}(\mathbf{k})$, for $\mathbf{k}=(0,1)$ and $(1,0)$, from Run D, with computed mean magnitudes of 8.46 and 10.30, respectively, differ from the expected mean magnitude of 9.50 (though the rms of the computed means is 9.42 ).

Our last figure, Fig. 9, draws on data from Run J, whose spectra are shown in Fig. 2. These spectra peak at $k^{2}=K^{2}=36,37$, so we look at the phase trajectories of $v^{(2)}(\mathbf{k})$, for $\mathbf{k}=(39,46)$ and $(-39,46)$ as representative examples. There appears to be significant structure in Fig. 9, though not as stationary as some of those seen in Figs. 5 and 6 for Run A, and Fig. 8 for Run D. In Fig. 9, the coefficients $v^{(2)}(\mathbf{k}), \mathbf{k}=(36,46)$ and $(-39,46)$, have mean magnitudes 60.4 and 56.1, respectively, while the expectation is 54.0. However, we could set our initial conditions so that the $v^{(1)}(\mathbf{k})$ have energies as small as desired, and in this way create essentially static structures in any run, whether their spectra peak at $k=1$ or at $k=K$.

We have now seen several examples of dynamical behavior that does not match ensemble expectations. We explain this anomalous behavior in the next section, using the concept of 


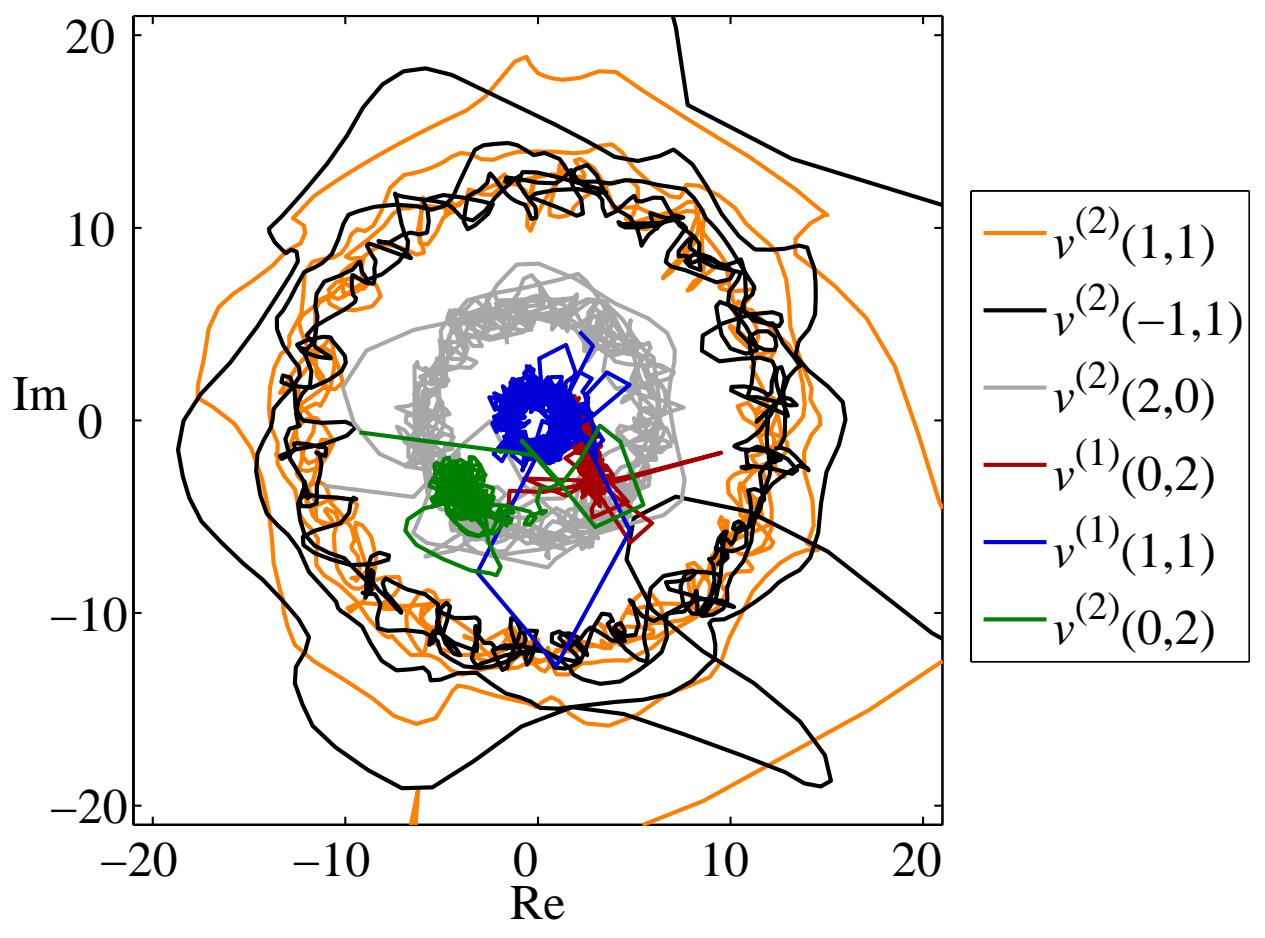

FIG. 6: Phase trajectories of $v^{(1,2)}(\mathbf{k}), k^{2}=2$ and 4, for Run A.

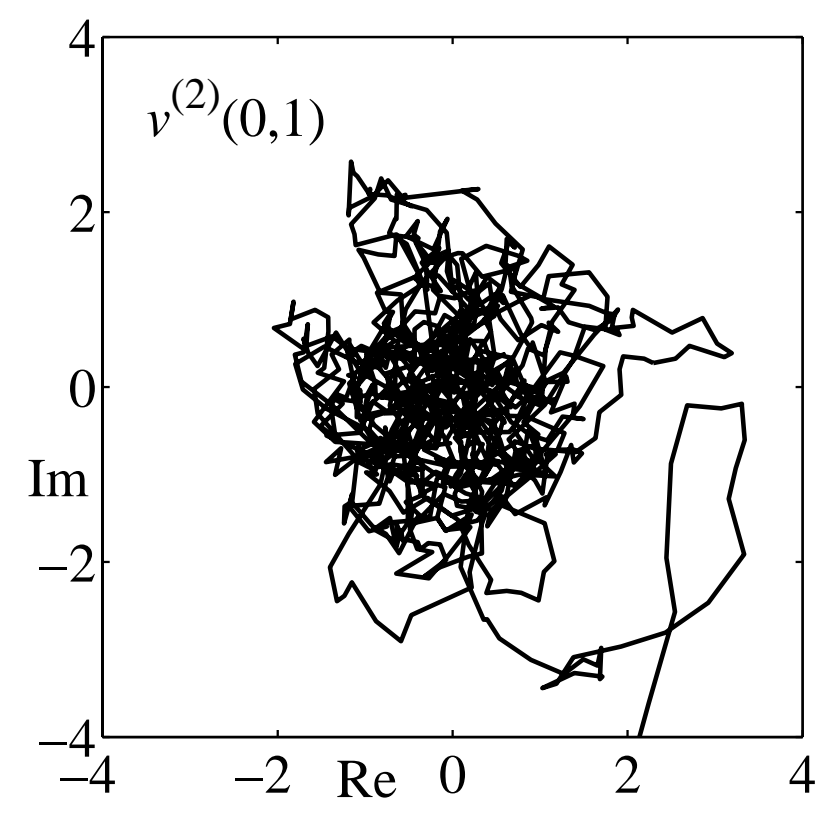

FIG. 7: Phase trajectory of $v^{(1)}(\mathbf{k}), \mathbf{k}=(0,1)$, for Run D. 


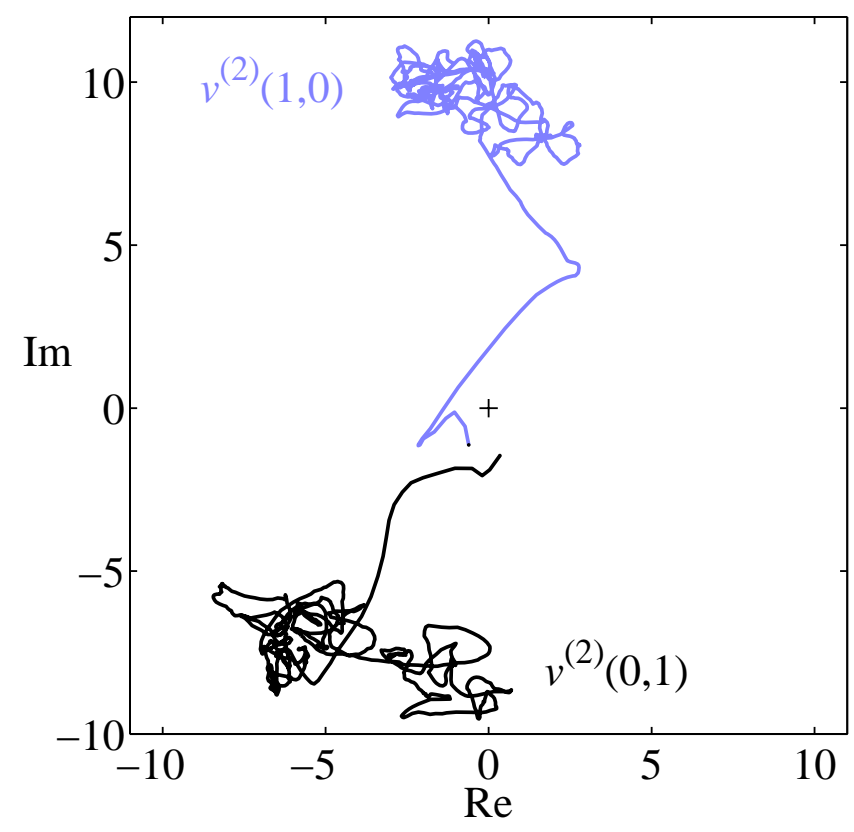

FIG. 8: Phase trajectories of the $v^{(2)}(\mathbf{k})$ with $k^{2}=1$ for Run D.

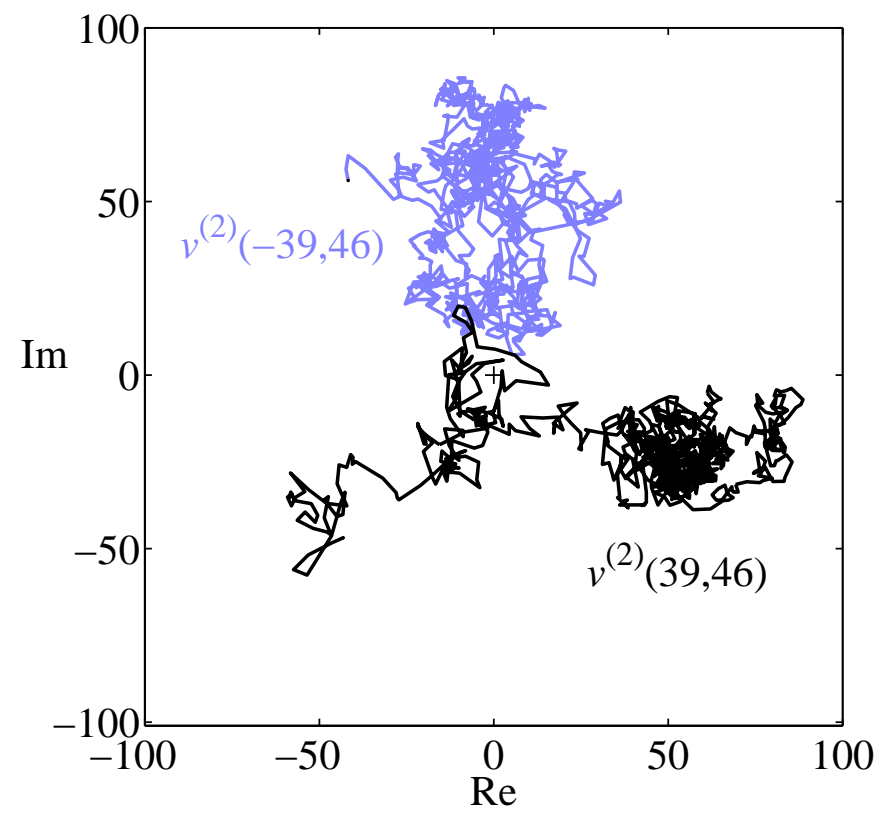

FIG. 9: Phase trajectory of $v^{(2)}(\mathbf{k}), \mathbf{k}=( \pm 39,46)$, for Run J.

broken ergodicity. We then discuss the implications for dissipative MHD turbulence. 


\section{BROKEN ERGODICITY}

The eigenvariables (38) are, again (with $C_{k}=\sqrt{\beta^{2}+\gamma^{2} / k^{4}}$ and $G_{k}=C_{k}-\gamma / k^{2}$ )

$$
\begin{aligned}
& \tilde{v}^{(1)}(\mathbf{k})=\frac{1}{\sqrt{2 C_{k} G_{k}}}\left[\frac{G_{k}}{k} \tilde{\omega}(\mathbf{k})+\beta k \tilde{a}(\mathbf{k})\right], \\
& \tilde{v}^{(2)}(\mathbf{k})=\frac{1}{\sqrt{2 C_{k} G_{k}}}\left[-\frac{\beta}{k} \tilde{\omega}(\mathbf{k})+G_{k} k \tilde{a}(\mathbf{k})\right] .
\end{aligned}
$$

As (45) shows, the expectation values $\left\langle\left|\tilde{v}^{(2)}(\mathbf{k})\right|^{2}\right\rangle$ are inversely proportional to the eigenvalues $\lambda_{k}^{(2)}(\mathbf{k})$, so that when certain eigenvalues $\lambda_{k}^{(2)}(\mathbf{k})$ are very small, the corresponding $\tilde{v}^{(2)}(\mathbf{k})$ can have very large magnitudes, as Fig. 2 shows, at either lowest or highest $k$, i.e., at $k=1$ or at $k=K$.

Whenever $\left\langle\left|\tilde{v}^{(2)}(\mathbf{k})\right|^{2}\right\rangle \gg\left\langle\left|\tilde{v}^{(1)}(\mathbf{k})\right|^{2}\right\rangle$, equations (69) and (70) can be approximated by

$$
\begin{gathered}
0 \approx \frac{1}{\sqrt{2 C_{k} G_{k}}}\left[\frac{G_{k}}{k} \tilde{\omega}(\mathbf{k})+\beta k \tilde{a}(\mathbf{k})\right], \\
\tilde{v}^{(2)}(\mathbf{k}) \approx \frac{1}{\sqrt{2 C_{k} G_{k}}}\left[-\frac{\beta}{k} \tilde{\omega}(\mathbf{k})+G_{k} k \tilde{a}(\mathbf{k})\right] .
\end{gathered}
$$

This leads to the approximate relationship $G_{k} \tilde{\psi}(\mathbf{k}) \approx-\beta \tilde{a}(\mathbf{k}) ;$ as $|\gamma| / k^{2} \rightarrow 0$, then $\tilde{\psi}(\mathbf{k}) \rightarrow$ $\pm \tilde{a}(\mathbf{k})$ and the corresponding modes no longer interact nonlinearly in the equations of motion (1) and (2).

In general, $\left\langle\left|\tilde{v}^{(2)}(\mathbf{k})\right|^{2}\right\rangle \gg\left\langle\left|\tilde{v}^{(1)}(\mathbf{k})\right|^{2}\right\rangle$ is best satisfied at either $k=1$ or $k=K$ and at these values of $k,\left\langle\left|\tilde{v}^{(2)}(\mathbf{k})\right|^{2}\right\rangle$ is much larger than for any other eigenmode (of the first or second kind) at any other values of $k$. Run A is an example of the largest expected eigenmode occurring at $k=1$, and Run $\mathrm{J}$ is an example of the largest expected eigenmode occurring at $k=K$, as is shown in Fig. 2. In these runs, once the dynamical system comes close to equilibrium, and eigenmode energies come close to their expectation values, then nonlinear interactions are greatly depressed and the time-evolution of the $\tilde{v}^{(2)}(\mathbf{k})$ for $k=1$ (Run A) or $k=K$ (Run J) slows down to a more or less stationary state, buffeted (slightly for Run A and more so for Run J) by what is effectively random noise. This process is seen in Fig. 5 for Run A, where $\tilde{v}^{(2)}(\mathbf{k})$ for $\mathbf{k}=(0,1)$ has reached a high degree of stationarity, and provides a 'mean magnetic field' on which the eigenmode $\tilde{v}^{(2)}(\mathbf{k})$ for $\mathbf{k}=(1,0)$ behaves like an Alfvén wave; further stationarity and Alfvén waves are seen in Fig. 6 for some $k=2$ 
and 4 eigenmodes in Run A. The process is also seen, to a lesser extent, in Fig. 8 for Run D, where the $\tilde{v}^{(2)}(\mathbf{k})$ for $k=1$ are quasi-staionary, and in Fig. 9 for Run J, where the $\tilde{v}^{(2)}(\mathbf{k})$ for $\mathbf{k}=( \pm 39,46)$, have reached rougher levels of stationarity. (Here, we have a numerical realization of the basic assumption from which Kraichnan started in his prediction of the inertial range energy spectrum for MHD turbulence ${ }^{26}$.)

This process, in which an eigenvalue becomes so small that (71) and (72) are fairly well satisfied and one or more eigenmodes become quasi-stationary, so that the expectation of zero mean for those eigenmodes is not met in a reasonable time, is the cause of broken ergodicity in numerical models of ideal, 2-D, homogeneous MHD turbulence. However, while broken ergodicity at $k=K$ is an interesting phenomenon in numerical models of ideal 2-D MHD turbulence, if $\nu$ and $\eta$ are nonzero in (1) and (2), then eigenmodes at or near $k=K$ will be highly dissipated, obviating the importance of any stationarity. In contrast, though dissipation is important at high $k$, it may have only minimal direct effects at low $k$ for large $N$, in which case we may expect that the ideal results presented here remain pertinent for real MHD turbulence.

Since $K$ may be made larger in 2-D simulations than in 3-D, work is underway to explore 2-D dissipative MHD turbulence on as large a grid as is practicable, in order to more fully investigate broken ergodicity in high Reynolds number MHD turbulence. Nevertheless, it is also important to continue 3-D studies, as there are quantitative and qualitative differences between 2-D and 3-D MHD turbulence. Ideal 3-D MHD turbulence exhibits broken ergodicity at $k=1$, but not at $k=2$ or 4 , though this may be due to the fact that long-term simulations ${ }^{10}$ have used $32^{3}$ grids, with $K=15.08$, while in the 2 -D simulations presented here, broken ergodicity appears at $k=1,2,4$, and higher, when $\widehat{\varphi}_{\mathrm{o}}>\frac{1}{2}$, but here $128^{2}$ grids were used, with $K=60.34$. Thus, still larger grids, which may more fully resolve the details of intrinsic coherent structure, are necessary for both 2-D and 3-D MHD turbulence simulations, remembering that very long simulation times are also required. The challenge is to find the most efficacious combination of constantly evolving computer software and hardware available for the task.

A major qualitative difference between ideal 2-D and 3-D MHD turbulence is that ideal spectra will peak at $k=K$ for $\widehat{\varphi}_{\mathrm{o}}<\frac{1}{2} 2$-D runs, while this cannot happen in ideal 3-D MHD simulations (expectation values of magnetic energy are never less than those for kinetic energy in ideal 3-D MHD turbulence ${ }^{28}$, but more pertinently, the smallest eigenvalues occur 
at only at $k=1$ for any expected value of magnetic energy ${ }^{10}$ ). However, there are enough similarities between 2-D and 3-D MHD turbulence that both merit continued study, both for their inherent mathematical interest and for their important applications.

\section{DISCUSSION}

Broken ergodicity in 2-D MHD turbulence depends on the approximations (71) and (72) being good ones that produce the relationship $G_{k} \tilde{\psi}(\mathbf{k}) \approx-\beta \tilde{a}(\mathbf{k})$ for either $k=1$ or $k=$ $K$. This relationship between $\tilde{\psi}(\mathbf{k})$ and $\tilde{a}(\mathbf{k})$ involves the cofactors $G_{k}$ and $\beta$ and thus depends explicitly on the inverse temperatures $\beta$ and $\gamma$. In contrast, analogous results for 3-D ideal MHD turbulence ${ }^{10}$ lead to relationships (but only at $k=1$ ) $\operatorname{such}$ as $\tilde{\mathbf{u}}(\hat{\mathbf{k}}) \approx \tilde{\boldsymbol{\omega}}(\hat{\mathbf{k}})$, $\tilde{\mathbf{j}}(\hat{\mathbf{k}}) \approx \tilde{\mathbf{b}}(\hat{\mathbf{k}})$ and $\tilde{\mathbf{u}}(\hat{\mathbf{k}}) \sim \tilde{\mathbf{b}}(\hat{\mathbf{k}})$, where $|\hat{\mathbf{k}}|=1$, that do not contain cofactors involving inverse temperatures. However, these relationships are similar in that they indicate that both 2-D and 3-D magnetofluids relax to 'force-free' states. When dissipation is present, higher $k$ modes decay quicker, so most of the energy eventually winds up in the $k=1$ modes, which, if these ideal results are pertinent, become force-free states of the maximum size permitted by boundary conditions.

Let us briefly place the matter into a historical context. In 1974, Taylor put forth the idea that a cylindrically confined plasma somehow relaxed to a state in which energy $E$ was minimized while magnetic helicity $H_{M}$ remained constant ${ }^{27}$. This led to a force-free configuration described by lowest-order Bessel functions. The qualitative process through which these Taylor states occur has been termed 'selective decay'29 or 'dynamic alignment'30, both of which were seen later to be synonymous with 'plasma relaxation'31. Related concepts are 'self-organization' in a plasma ${ }^{32}$ and 'depression of nonlinearity'33,34. 'Broken ergodicity', in turn, provides a quantitative, mathematical explanation for the process of large-scale structure formation, dynamo action and self-organization in model systems describing ideal, homogeneous MHD turbulence. The addition of dissipation to these model systems has been seen through numerical simulation ${ }^{16,28}$ to lead to selective decay and dynamical alignment, while the quasi-stationary large-scale structures due to broken ergodicity were still in evidence. Although these simulations were on only moderately sized grids, we fully expect to see even more compelling evidence as future long-time simulations that use larger and larger sized grids become possible. 


\section{CONCLUSION}

In summary, we have explained previous observations of coherent structure and apparent non-ergodicity in numerical simulations of 2-D MHD turbulence as being due to broken ergodicity. As stated earlier, the next step is to perform dissipative simulations on as large a grid as practicable, to see how ideal results translate over to more realistic model systems. Keeping these simulations in a 2-D framework allows us to keep the maximum wavenumber $K$, as well as the dissipation wavenumber $k_{D}<K$, as large as possible. The results presented here indicate that there is a rich and complex nonlinear dynamics inherent in the model systems that represent 2-D MHD turbulence, and further study should reveal even more detail.

Advances in 3-D simulations are, perhaps, of more practical importance as these simulations are more directly related to understanding turbulent plasmas in both laboratory and astrophysical settings. However, since maximum wavenumbers $K$ are always going to be greater in 2-D simulations, the higher Reynolds numbers that are possible will continue to make 2-D simulations attractive for the study of MHD turbulence, notwithstanding the inherent difference between the 2-D and 3-D cases. The increasing capability of computer systems with regard to size and speed will allow us to gain even more insight into both 2-D and 3-D MHD turbulence, and we look forward to learning of new results in both cases.

1. D. Biskamp, Magnetohydrodynamic Turbulence (Cambridge U. P., UK, 2003).

2. L. D. Landau and E. M. Lifshitz, Fluid Mechanics (2nd Ed., Pergamon, Oxford, 1987), pp. 129-135.

3. W. Heisenberg, Proc. Roy. Soc. Lond. A 195, 402-406 (1948).

4. A. S. Monin and A. M. Yaglom, Statistical Fluid Mechanics: Mechanics of Turbulence, Vols. I and II (Dover, New York, 2007).

5. U. Frisch, A. Pouquet, J. Leorat, and A. Mazure, J. Fluid Mech. 68, 769-778 (1975).

6. D. Fyfe and D. Montgomery, J. Plasma Phys. 16, 181-191 (1976).

7. J. V. Shebalin, W. H. Matthaeus and D. Montgomery, J. Plasma Phys. 29, 525-547 (1983).

8. R. G. Palmer, Adv. Phys. 31, 669-735 (1982).

9. J. V. Shebalin, Physica D 37, 173-191 (1989). 
10. J. V. Shebalin, Phys. Plasmas. 16, 072301-1-14 (2009).

11. W. H. Matthaeus, W. T. Stribling, D. Martinez, S. Oughton and D. Montgomery, Physica D 51, 531-538 (1991).

12. D. Montgomery, W. H. Matthaeus, W. T. Stribling, D. Martinez and S. Oughton, Phys. Fluids A 4, 3-6 (1992).

13. D. Balsara and A. Pouquet, Phys. Plasmas 6, 89-99 (1999).

14. D. C. Robinson and M. G. Rusbridge, Phys. Fluids 14, 2499-2511 (1971).

15. S. J. Zweben, C. R. Menyuk and R. J. Taylor, Phys. Rev. Lett. 42, 1270-1274 (1979).

16. J. V. Shebalin, Discrete Contin. Dyn. Syst. B 5, 153-175 (2005).

17. S. A. Orszag and G. S. Patterson, Phys. Rev. Lett. 28, 76-79 (1972).

18. P. K. Newton, The N-Vortex problem: analytical techniques (Springer-Verlag, NY, 2001).

19. G. Joyce and D. Montgomery, J. Plasma Phys. 10, 107-121 (1973).

20. G. S. Patterson and S. A. Orszag, Phys. Fluids 14, 2538-2541 (1971).

21. J. Gazdag, J. Comp. Phys. 20, 196-207 (1976).

22. T. D. Lee, Q. Appl. Math. 10, 69 (1952).

23. W. M. Elsässer, Rev. Mod. Phys. 28, 135-163 (1956).

24. A. I. Khinchin, Mathematical Foundations of Statistical Mechanics (Dover, New York, 1949), pp. $137-145$.

25. J. V. Shebalin, J. Plasma Phys. 56, 419-426 (1996).

26. R. H. Kraichnan, Phys. Fluids 8, 1385-1387 (1965).

27. J. B. Taylor, Phys. Rev. Lett. 33, 1139-1141 (1974).

28. J. V. Shebalin, Phys. Plasmas 14, 102301 (2007).

29. W. H. Matthaeus and D. Montgomery, "Selective Decay Hypothesis at High Mechanical and Magnetic Reynolds Numbers," in Nonlinear Dynamics, edited by R. H. G. Helleman (New York Acad. Sci. New York, 1980) pp. 203-222.

30. W. H. Matthaeus, D. Montgomery and M. L. Goldstein, Phys. Rev. Lett. 51, 1484-1487 (1983).

31. A. C. Ting, W. H. Matthaeus and D. Montgomery, Phys. Fluids 29, 3261-3274 (1986).

32. B. B. Kadomtsev, Radiophys. Quant. Electron. 29, 781-787 (1986).

33. R. H. Kraichnan and R. Panda, Phys. Fluids 31, 2395-2397 (1988).

34. S. Servidio, W. H. Matthaeus and P. Dmitruk, Phys. Rev. Lett. 100, 095005 (2008). 